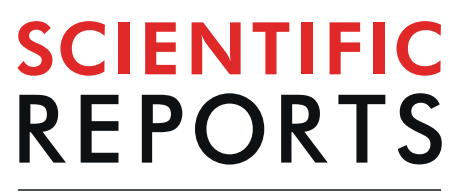

natureresearch

\title{
OncoOmics approaches to reveal essential genes in breast cancer: a panoramic view from pathogenesis to precision medicine
}

Andrés López-Cortés 1,2,3* César Paz-y-Miño ${ }^{1}$ Santiago Guerrero 1 Alejandro CabreraAndrade $^{2,4,5}$, Stephen J. Barigye ${ }^{6}$, Cristian R. Munteanu ${ }^{2,7,8}$, Humberto González-Díaz ${ }^{9,10}$, Alejandro Pazos ${ }^{2,7,8}$, Yunierkis Pérez-Castillo $\mathbb{1}^{5,11}$ \& Eduardo Tejera ${ }^{5,12^{*}}$

Breast cancer (BC) is the leading cause of cancer-related death among women and the most commonly diagnosed cancer worldwide. Although in recent years large-scale efforts have focused on identifying new therapeutic targets, a better understanding of $\mathrm{BC}$ molecular processes is required. Here we focused on elucidating the molecular hallmarks of $\mathrm{BC}$ heterogeneity and the oncogenic mutations involved in precision medicine that remains poorly defined. To fill this gap, we established an OncoOmics strategy that consists of analyzing genomic alterations, signaling pathways, protein-protein interactome network, protein expression, dependency maps in cell lines and patient-derived xenografts in 230 previously prioritized genes to reveal essential genes in breast cancer. As results, the OncoOmics BC essential genes were rationally filtered to 140 . mRNA up-regulation was the most prevalent genomic alteration. The most altered signaling pathways were associated with basal-like and Her2-enriched molecular subtypes. RAC1, AKT1, CCND1, PIK3CA, ERBB2, CDH1, MAPK14, TP53, MAPK1, SRC, RAC3, $B C L 2, C T N N B 1, E G F R, C D K 2, G R B 2, M E D 1$ and GATA3 were essential genes in at least three OncoOmics approaches. Drugs with the highest amount of clinical trials in phases 3 and 4 were paclitaxel, docetaxel, trastuzumab, tamoxifen and doxorubicin. Lastly, we collected $\sim 3,500$ somatic and germline oncogenic variants associated with 50 essential genes, which in turn had therapeutic connectivity with 73 drugs. In conclusion, the OncoOmics strategy reveals essential genes capable of accelerating the development of targeted therapies for precision oncology.

Breast cancer $(\mathrm{BC})$ is a complex and heterogeneous disease characterized by an intricate interplay between different biological aspects such as ethnicity, genomic alterations, gene expression deregulation, hormone disruption, signaling pathway alterations, hypoxia, and environmental determinants ${ }^{1,2}$. Over the last years, prevention, treatment and survival strategies have evolved favorably; however, there are BC profiles that remain incurable ${ }^{3}$.

${ }^{1}$ Centro de Investigación Genética y Genómica, Facultad de Ciencias de la Salud Eugenio Espejo, Universidad UTE, Mariscal Sucre Avenue, Quito, 170129, Ecuador. ${ }^{2}$ RNASA-IMEDIR, Computer Science Faculty, University of A Coruna, A Coruna, 15071, Spain. ${ }^{3}$ Red Latinoamericana de Implementación y Validación de Guías Clínicas Farmacogenómicas (RELIVAF-CYTED), Quito, Ecuador. ${ }^{4}$ Carrera de Enfermería, Facultad de Ciencias de la Salud, Universidad de Las Américas, Avenue de los Granados, Quito, 170125, Ecuador. ${ }^{5}$ Grupo de Bio-Quimioinformática, Universidad de Las Américas, Avenue de los Granados, Quito, 170125, Ecuador. ${ }^{6}$ Department of Chemistry, McGill University, 801 Sherbrooke Street West, Montreal, OC, H3A 0B8, Canada. ${ }^{7}$ Biomedical Research Institute of A Coruña (INIBIC), University Hospital Complex of A Coruna (CHUAC), A Coruna, 15006, Spain. ${ }^{8}$ Centro de Investigación en Tecnologías de la Información y las Comunicaciones (CITIC), Campus de Elviña s/n, A Coruna, 15071, Spain. ${ }^{9}$ Department of Organic Chemistry II, University of the Basque Country UPV/EHU, Leioa, 48940, Biscay, Spain. ${ }^{10}$ IKERBASOUE, Basque Foundation for Science, Bilbao, 48011, Biscay, Spain. ${ }^{11}$ Escuela de Ciencias Físicas y Matemáticas, Universidad de Las Américas, Avenue de los Granados, Quito, 170125, Ecuador. ${ }^{12}$ Facultad de Ingeniería y Ciencias Agropecuarias, Universidad de Las Américas, Avenue de los Granados, Quito, 170125, Ecuador. *email: aalc84@ gmail.com; eduardo.tejera@udla.edu.ec 
Nowadays, BC is the leading cause of cancer-related death among women $(627,000 ; 15 \%$ cases $)$ and the most commonly diagnosed cancer $(2,088,849 ; 24 \%$ cases $)$ worldwide ${ }^{4}$.

The development of large-scale DNA sequencing, gene expression, proteomics, large-scale RNA interference (RNAi) screens, large-scale CRISPR-Cas9 screens and patient-derived xenografts (PDXs) has allowed us to better understand the molecular landscape of oncogenesis. Considerable progress has been made in discovering coding and non-coding somatic drivers ${ }^{5,6}$, cancer driver genes ${ }^{7,8}$, cancer driver mutations ${ }^{9,10}$, germline variants ${ }^{11}$, driver fusion genes ${ }^{12,13}$, alternatively spliced transcripts ${ }^{14}$, expression-based stratification ${ }^{15}$, molecular subtyping ${ }^{16}$, biomarkers ${ }^{17}$, druggable enzymes ${ }^{18}$, cancer dependencies ${ }^{19-22}$, and drug resistance ${ }^{23}$.

Scientific advances made to date mark the era called the "end of the beginning" of cancer omics. In other words, each approach that was previously mentioned needs to be fully understood as a part of a complex network, analyzing the mechanistic interplay of signaling pathways, protein-protein interactome (PPi) networks, enrichment maps, gene ontology (GO), deep learning, molecular dependencies and genomic alterations per intrinsic molecular subtype: basal-like (estrogen receptor $(\mathrm{ER})^{-}$, progesterone receptor $(\mathrm{PR})^{-}$, human epidermal growth factor receptor $2(\mathrm{Her} 2)^{-}$, cytokeratin $5 / 6^{+}$and/or $\left.\mathrm{EGFR}^{+}\right)$; Her2-enriched $\left(\mathrm{ER}^{-}, \mathrm{PR}^{-}, \mathrm{Her} 2^{+}\right)$; luminal A $\left(\mathrm{ER}^{+}\right.$ and/or $\mathrm{PR}^{+}$, Her2 ${ }^{-}$, low Ki67); luminal B with Her2 ${ }^{-}\left(\mathrm{ER}^{+}\right.$and/or $\mathrm{PR}^{+}, \mathrm{Her}^{-}$, low Ki67); luminal B with Her2 ${ }^{+}$ $\left(\mathrm{ER}^{+}\right.$and/or $\mathrm{PR}^{+}, \mathrm{Her}^{+}$, any Ki67); and normal like ${ }^{24-30}$.

Here we focus on elucidating the molecular hallmarks of BC essential genes and the oncogenic mutations applied in precision medicine that remains poorly defined. To fill this gap, we propose the OncoOmics strategy that consists in the analysis of genomic alterations (mRNA up-regulation, mRNA down-regulation, putative driver mutation, copy number variant (CNV) amplification, CNV deep deletion, and fusion gene), signaling pathways, PPi network, protein expression, BC dependencies in cell lines and patient-derived xenografts in a set of previously prioritized genes. These genes will come from our Consensus Strategy (CS) study ${ }^{29}$, the Pan-Cancer Atlas (PCA) project ${ }^{3,13,31-37}$, the Cancer Genome Interpreter (CGI) study ${ }^{38}$, and the Pharmacogenomics Knowledgebase (PharmGKB) ${ }^{39}$.

In our previous studies, López-Cortés et al., Tejera et al., and Cabrera-Andrade et al., developed a Consensus Strategy that was proved to be highly efficient in the recognition of gene-disease association ${ }^{29,40,41}$. The main objective was to apply several bioinformatics methods to explore BC pathogenic genes. On the other hand, The Cancer Genome Atlas (TCGA) has concluded the most sweeping cross-cancer analysis yet undertaken, namely the PCA project ${ }^{32}$. PCA reveals how genomic alterations and protein expression collaborate in BC progression, providing insights to prioritize the development of new treatments $\mathrm{s}^{3,13,31-37}$. The CGI flags genomic biomarkers of drug response with different levels of clinical relevance ${ }^{38}$. Lastly, PharmGKB is a comprehensive resource that curates and spreads knowledge of the impact of clinical annotations on drug respons $\mathrm{e}^{39,42}$. PharmGKB collects the precise guidelines for the application of precision medicine and pharmacogenomics in clinical practice published by the European Society for Medical Oncology (ESMO), the National Comprehensive Cancer Network (NCCN), the Royal Dutch Association for the Advancement of Pharmacy (DPWG), the Canadian Pharmacogenomics Network for Drug Safety (CPNDS) and the Clinical Pharmacogenetics Implementation Consortium (CPIC) $)^{43-46}$. Hence, we identified essential genes, oncogenic mutations and potential therapeutic targets that could be incorporated into strategies aimed at improving novel drug development and precision medicine in BC.

\section{Results}

OncoPrint of genomic alterations according to the Pan-Cancer Atlas. PCA has reported the clinical data of 1084 individuals with BC and it can be visualized in the Genomic Data Commons of the National Cancer Institute and in the $\mathrm{cBioPortal}^{47,48}$. In regard to molecular subtypes and tumor stages, $46 \%$ were lumina A, $18 \%$ luminal B, $7 \%$ Her2-enriched, $16 \%$ basal-like and $3 \%$ normal-like, whereas $17 \%$ were tumor stage 1 (T1), 58\% T2 stage, 23\% T3 stage and 2\% T4 stage (Supplementary Table S1).

Figure la shows the frequency mean of genomic alterations per gene set. The frequency mean of the PCA gene set was 1.3, followed by the CS gene set (1.2), the PharmGKB/CGI gene set (1.0), BC driver genes (0.8), and non-cancer genes (0.4) (Supplementary Table S2). Consequently, we performed a multiple comparison of the genomic alteration frequencies using the Bonferroni correction in order to determine statistical significance among gene sets. There were significant differences between $\mathrm{BC}$ driver genes and non-cancer genes $(\mathrm{P}<0.001)$, the PCA gene set and $\mathrm{BC}$ driver genes $(\mathrm{P}<0.001)$, and the $\mathrm{CS}$ gene set and $\mathrm{BC}$ driver genes $(\mathrm{P}<0.001)$. Hence, the fact that gene sets of interest (CS and PCA) presented significant differences in the amount of genomic alterations versus BC driver genes could indicate that we are analyzing potentially essential genes in BC. Figure $1 \mathrm{~b}$ shows the percentage of genomic alterations per type. The most common genomic alterations were mRNA up-regulation (55.8\%), CNV amplification (17.1\%), and missense mutations (8.4\%). Figure 1c shows the ratio of genomic alterations in the 230 genes per sample and molecular subtype. Basal-like had the highest ratio $(n=33)$, followed by Her2-enriched (29), luminal B (24), normal-like (17), and luminal A (15). The ratio of all BC samples was 19.6. Figure $1 \mathrm{~d}$ shows the ratio of genomic alterations in the 230 genes per sample and tumor stage. T2 stage had the highest ratio (23), followed by T3 (22), T1 (17) and T4 (8). Figure 1e,f show the percentage of genomic alterations per subtype and tumor stage, respectively. mRNA up-regulation and CNV amplification were the most common alterations in all molecular subtypes and tumor stages.

Figure 2 shows the ranking of genes with the highest amount of genomic alterations per molecular subtype and tumor stage. Regarding molecular subtypes, PIK3CA was the most altered gene in luminal A, CCND1 in luminal B, TP53 in basal-like and normal-like, and ERBB2 in Her2-enriched (Fig. 2a). Figure 2b-f show genes with the highest ratio of mutations, CNV amplifications, CNV deep deletions, mRNA up-regula tions, and mRNA down-regulations per molecular subtype (Tables S3-S7). After Bonferroni correction, we obtained statistically significant differences $(\mathrm{P}<0.05)$ regarding $\mathrm{CNV}$ amplifications, $\mathrm{CNV}$ deep deletions, $\mathrm{mRNA}$ up-regulations, and mRNA down-regulations among molecular subtypes. On the other hand, the most altered genes per tumor stage were PIK3CA in T1 stage, TP53 in T2 and T3, and ERBB2 in T4 (Fig. 2g). Figure 2h-1 show genes with the 
a
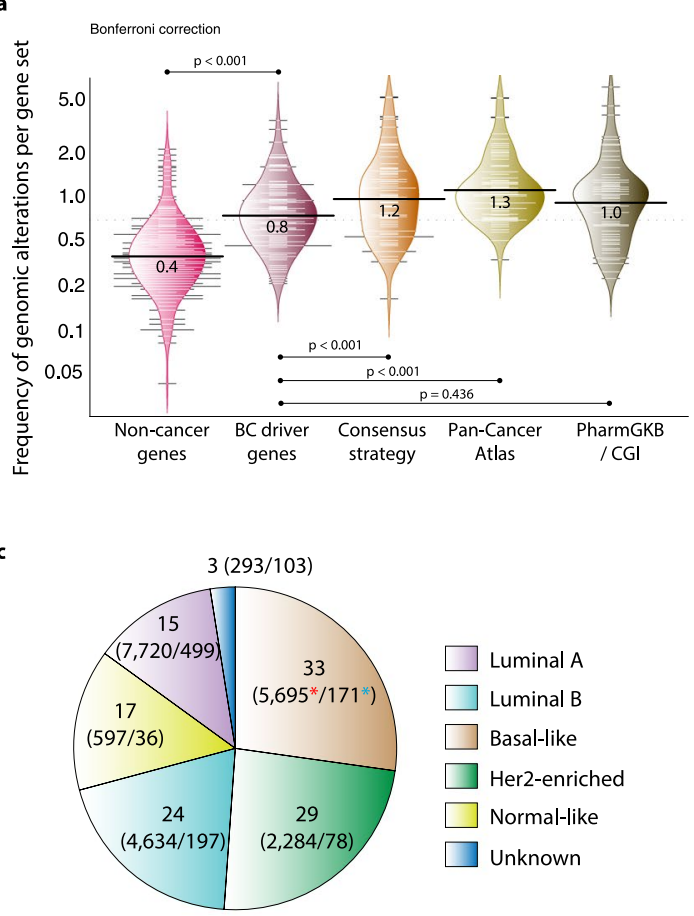

Genomic alterations* (230 genes) per sample* and per intrinsic molecular subtype (mutation, CNV, fusion and mRNA)

Total ratio: $19.6(21,223 * / 1,084 *)$

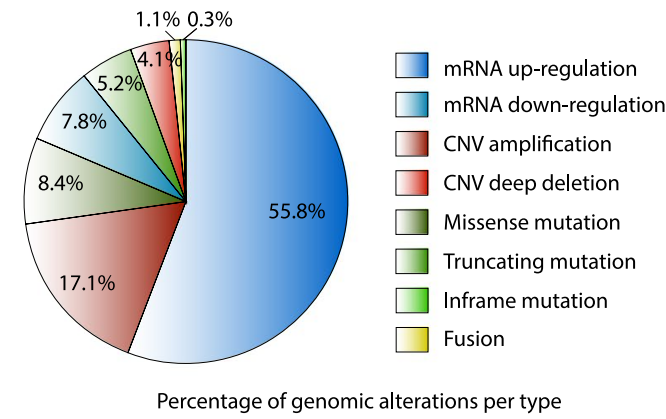

d

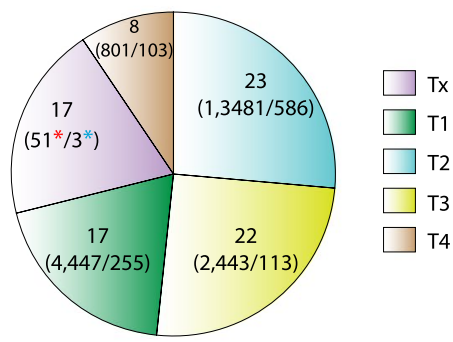

Genomic alterations* (230 genes) per sample* and per tumor stage

Total ratio: $20\left(21,223 * / 1,060^{*}\right)$ e

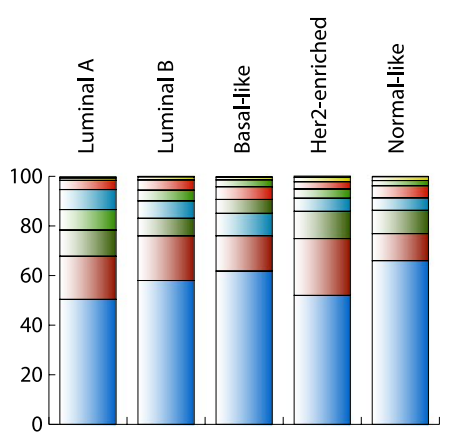

Percentage of genomic alterations per subtype

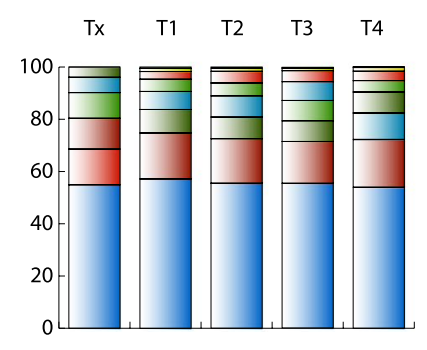

Percentage of genomic alterations per tumor stage

Figure 1. Genomic alterations of the breast cancer cohort according to PCA. (a) Frequency of genomic alterations per gene set (non-cancer genes, BC driver genes according to the Network of Cancer Genes, Consensus Strategy, BC genes according to PCA, BC biomarkers according to the PharmGKB and CGI). Bonferroni correction with significant level of $\mathrm{P}<0.05$ and a $95 \%$ confidence interval was performed. (b) Percentage of genomic alterations per type. (c) Ratio of genomic alterations per intrinsic molecular subtype. (d) Ratio of genomic alterations per tumor stage. (e) Percentage of genomic alterations per type and molecular subtype. (f) Percentage of genomic alterations per type and tumor stage.

highest percentage of mutations, $\mathrm{CNV}$ amplifications, CNV deep deletions, mRNA up-regulations, and mRNA down-regulations per tumor stage (Tables S8-S12). We found statistically significant differences $(\mathrm{P}<0.05)$ regarding all genomic alterations among tumor stages using the Bonferroni correction test.

The first OncoOmics approach was focused on genes with the highest amount of genomic alterations (more than the average). The panoramic landscape of genomic alterations was termed OncoPrint and is shown in Fig. $3 \mathrm{a}$. Putative driver mutations were taken into account for this analysis, discarding passenger mutations (Figure S1 and Supplementary Table S13). Figure 3b,c show circos plots of interactions among molecular subtypes, tumor stages, and genomic alterations of the most altered genes (Supplementary Table S14). Highest amount of fusion genes were in Her2-enriched subtype and T4 stage, highest amount of mRNA down-regulation + CNV deep deletion were in basal-like subtype and T4 stage, highest amount of mRNA up-regulation + CNV amplification were in basal-like subtype and T4 stage, lastly, highest amount of putative driver mutations were in Her2-enriched subtype and T3 stage. As result, the first OncoOmics approach reveled 73 essential genes with highest frequencies of genomic alterations. 
a * Ratio of genomic alterations per sample and per intrinsic molecular subtype

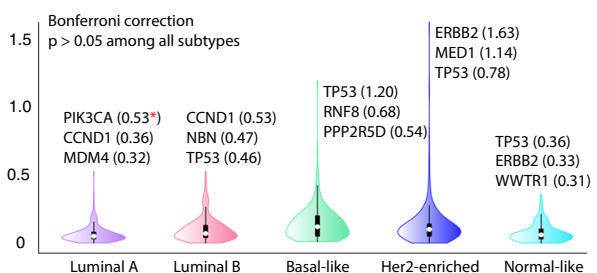

b Punctual mutations (mutation + fusion)

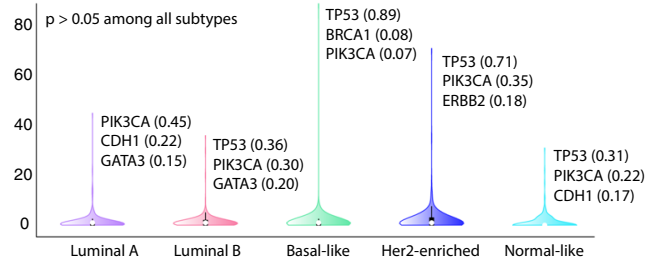

C CNV amplification

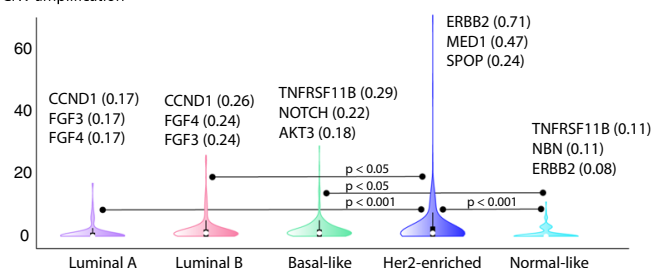

d CNV deep deletion

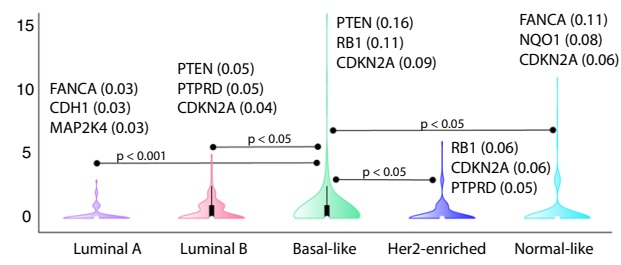

e mRNA up-regulation

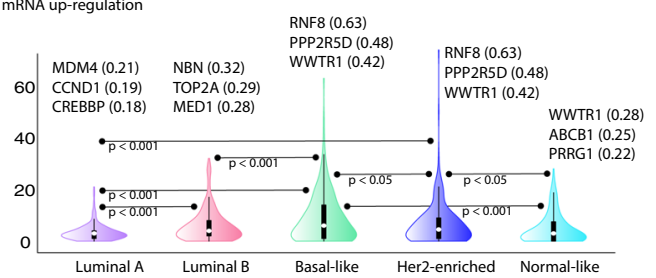

f mRNA down-regulation

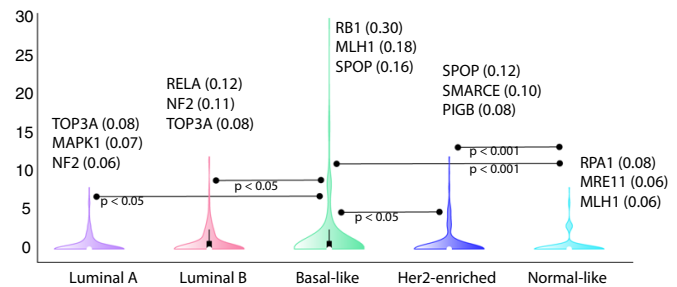

g * Ratio of all genomic alterations per sample and per tumor stage

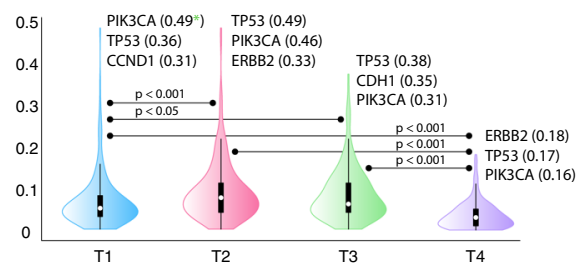

h Punctual mutations (mutation + fusion

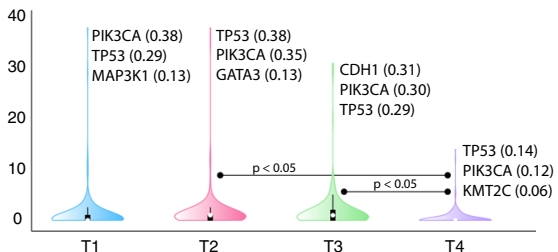

i CNV amplification

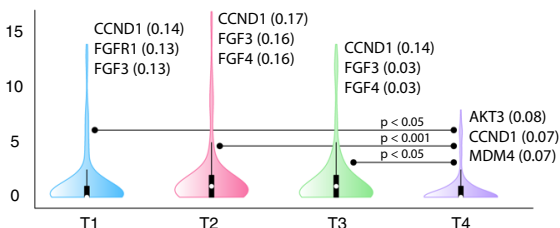

j CNV deep deletion

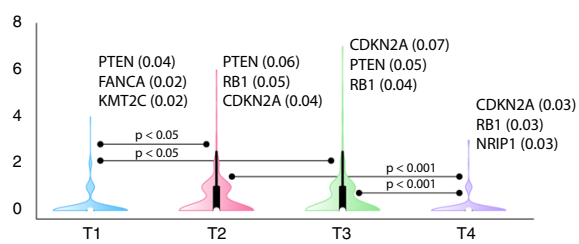

k mRNA up-regulation

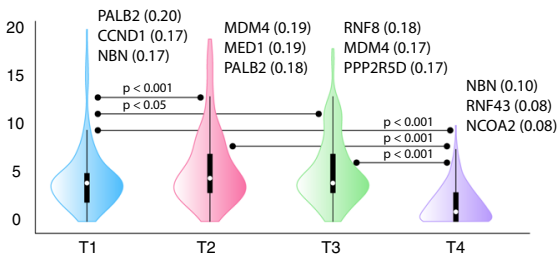

I mRNA down-regulation

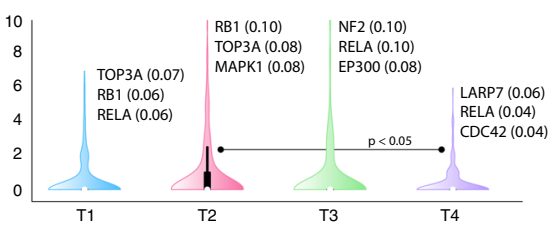

Figure 2. Ranking of genes with the highest amount of genomic alterations per molecular subtype and tumor stage. (a) Frequency of genomic alterations (punctual mutations, copy number variants and mRNA expression) per molecular subtype. (b) Frequency of genomic alterations per tumor stage. (c) Frequency of punctual mutations per molecular subtype. (d) Frequency of punctual mutations per tumor stage. (e) Frequency of CNV amplifications per molecular subtype. (f) Frequency of CNV amplifications per tumor stage. (g) Frequency of CNV deep deletions per molecular subtype. (h) Frequency of CNV deep deletions per tumor stage. (i) Frequency of mRNA up-regulation per molecular subtype. (j) Frequency of mRNA up-regulation per tumor stage. (k) Frequency of mRNA down-regulation per molecular subtype. (L) Frequency of mRNA downregulation per tumor stage. 
a

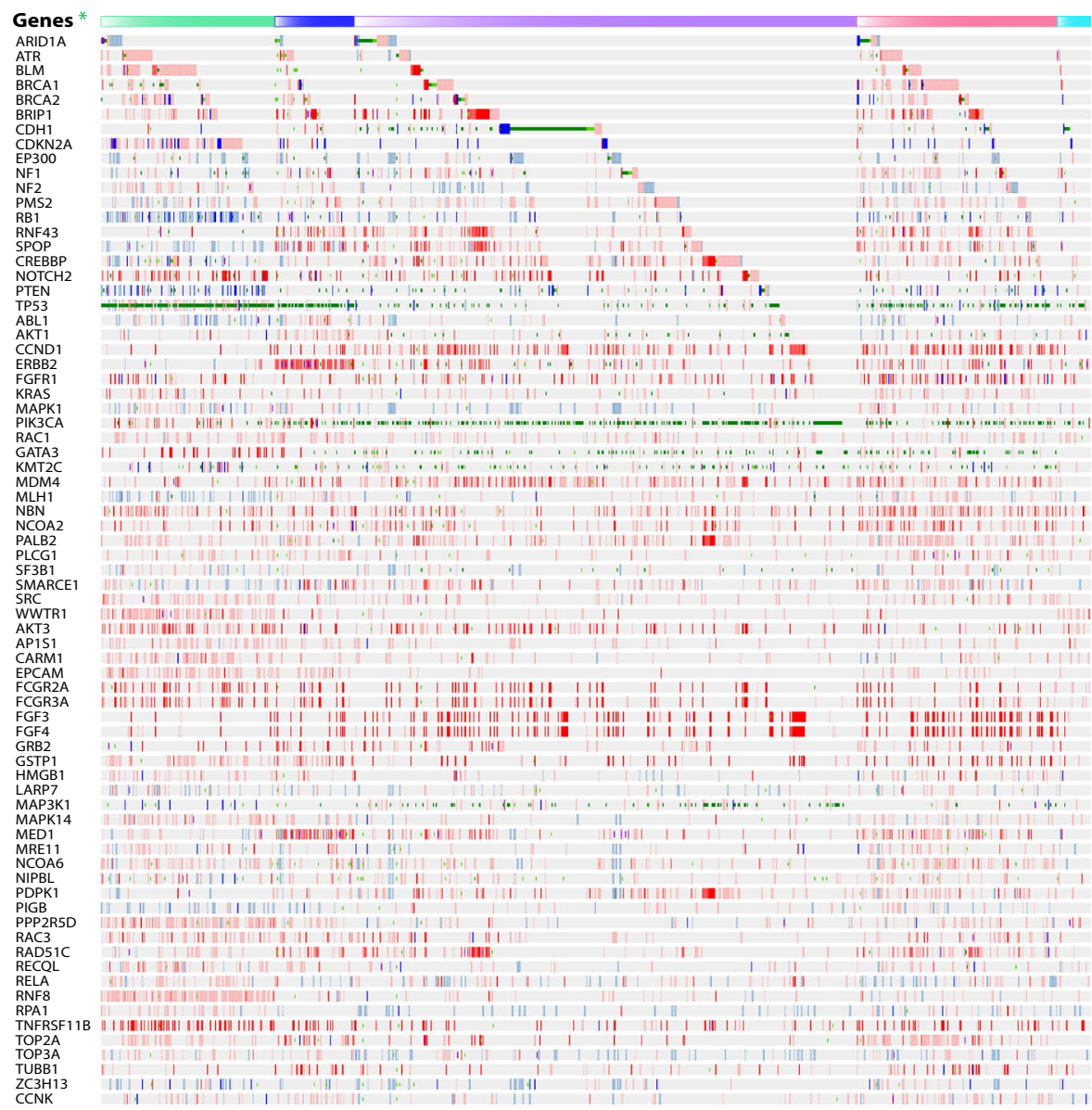

Genomic alterations

- Putative driver mutations

- Mutation (unknown significance)

- Fusion

CNV amplification

CNV deep deletion

mRNA up-regulation

mRNA down-regulation

Intrinsic molecular subtype

Basal-like

Her2-enriched

Luminal $A$

1 Luminal B

II Normal-like b

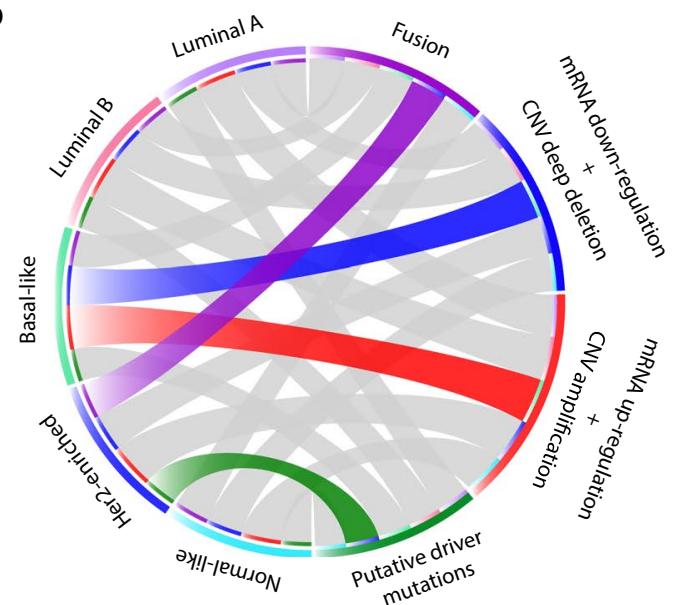

C

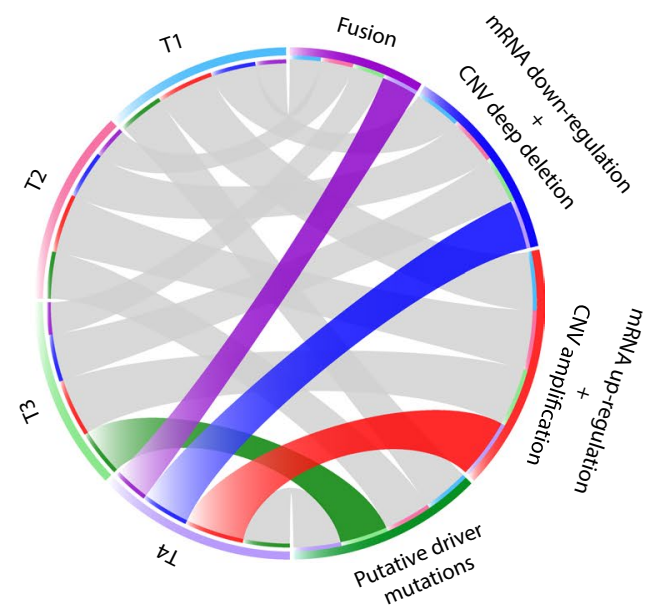

Figure 3. OncoPrint of genomic alterations according to the Pan-Cancer Atlas. (a) OncoPrint of genes with more genomic alterations than the average ( $>86)$ per molecular subtype. (b) Circos plot between molecular subtypes and the highest amount of genomic alterations (fusion genes, mRNA down-regulation plus CNV deep deletion, mRNA upregulation plus CNV amplification, and driver mutations). (c) Circos plot between tumor stages and the highest amount of genomic alterations.

Pathway enrichment analysis. This enrichment analysis was performed using David Bioinformatics Resource to obtain integrated information from the Kyoto Encyclopedia of Genes and Genomes (KEGG ${ }^{49-52}$. The enrichment analysis of signaling pathways was carried on in the 230 genes, obtaining more than 50 terms with a Benjamini-Hochberg - false discovery rate (FDR) $<0.01$ (Supplementary Table S15). Subsequently, genomic alterations of genes that make up each signaling pathway were analyzed according to the molecular subtype and tumor stage. Figure 4a shows a circos plot correlating molecular subtypes with signaling pathways 


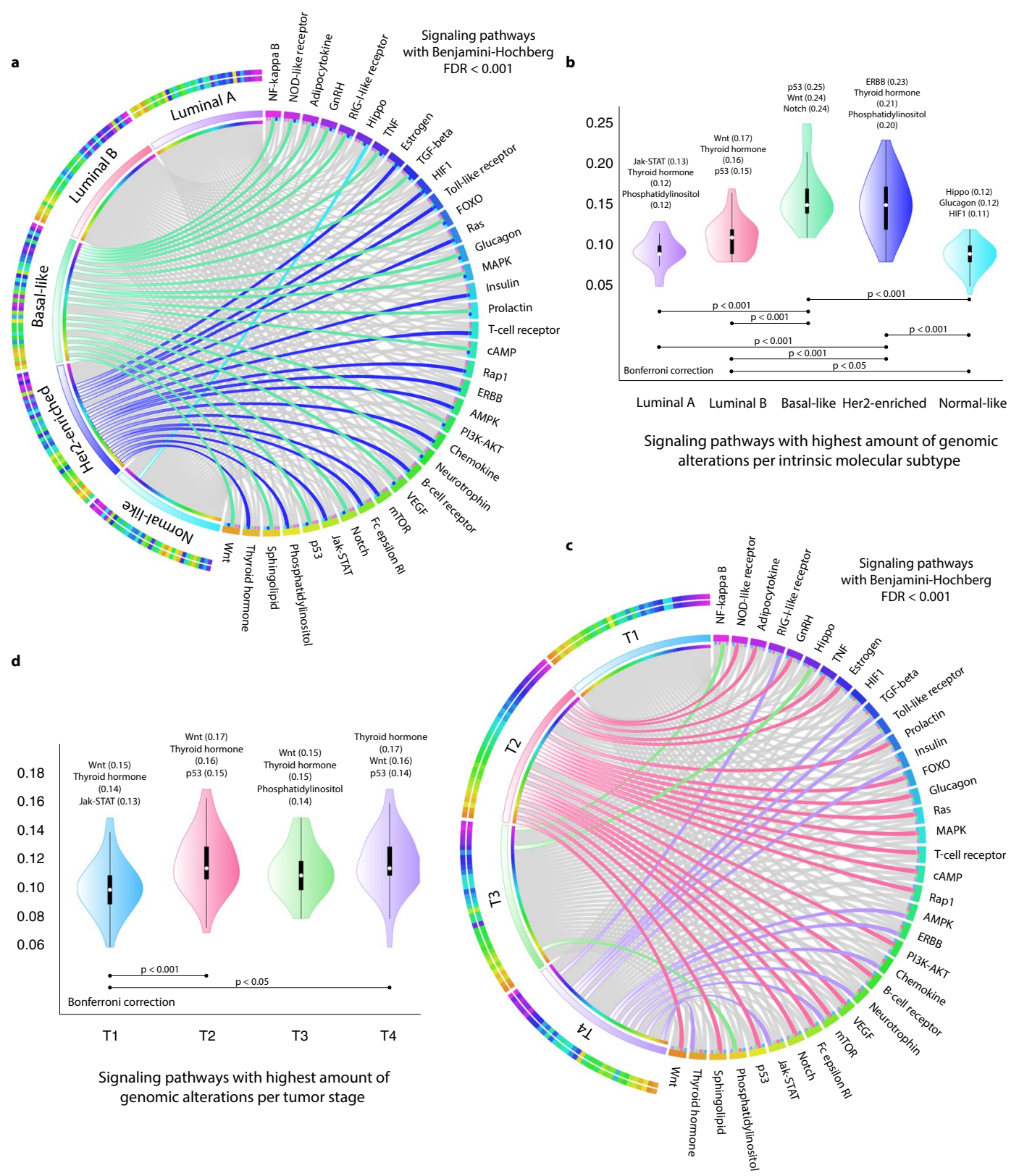

Figure 4. Pathway enrichment analysis per molecular subtype and tumor stage. (a) Circos plot between molecular subtypes and the most altered signaling pathways. (b) Violin plots showing the frequency of the most altered signaling pathways per molecular subtype. (c) Circos plot between tumor stages and the most altered signaling pathways. (d) Violin plots showing the frequency of the most altered signaling pathways per tumor stage.

(Supplementary Table S16). NF-kappa ß, NOD-like receptor, adipocytokine, GnRH, RIG-like receptor, TNF, TGFß, FOXO, glucagon, MAPK, prolactin, cAMP, PI3K-AKT, neurotrophin, VEGF, notch, p53, sphingolipid and Wnt signaling pathways were more altered in basal-like; estrogen, HIF1, toll-like receptor, ras, insulin, T-cell receptor, rap1, ERBB, AMPK, chemokine, B-cell receptor, mTOR, Fc-epsilon RI, Jak-STAT, phosphatidylinositol and thyroid hormone pathways were more altered in Her2-enriched; and Hippo pathway in normal-like. On the other hand, Fig. $4 \mathrm{~b}$ shows the ranking of the most altered signaling pathways per molecular subtype. Jak-STAT pathway was more altered in luminal A; Wnt pathway in luminal B; p53 pathway in basal-like; ERBB pathway in Her2-enriched; and Hippo pathway in normal-like (Supplementary Table S17). After Bonferroni correction, we observed statistically significant differences $(\mathrm{P}<0.001)$ regarding the amount of genomic alterations in signaling pathways among molecular subtypes.

Figure $4 \mathrm{c}$ shows a circos plot correlating tumor stages with signaling pathways according to the frequency of genomic alterations (Supplementary Table S16). NOD-like receptor, adipocytokine, GnRH, TNF, estrogen, prolactin, FOXO, glucagon, ras, MAPK, T-cell receptor, cAMP, rap1, PI3K-AKT, B-cell receptor, VEGF, mTOR, Fc 
epsilon RI, NOTCH, p53, sphingolipid and Wnt pathways were more altered in stage T2; NF-kappa ß, Hippo and phosphatidylinositol pathways were more altered in T3 stage; and RIG-like receptor, HIF1, TGFß, toll-like receptor, insulin, AMPK, ERBB, chemokine, neurotrophin, mTOR, jak-STAT and thyroid hormone pathways were more altered in T4 stage. On the other hand, Fig. 4d shows the ranking of the most altered signaling pathways per tumor stage. Wnt pathway was more altered in T1, T2 and T3 stages; and thyroid hormone pathway was more altered in T4 stage (Supplementary Table S18). We found statistically significant differences $(\mathrm{P}<0.001)$ regarding the amount of genomic alterations in signaling pathways among different tumor stages using the Bonferroni correction test.

Protein-protein interactome network. The second OncoOmics approach was focused on proteins with the highest degree centrality and consensus score in the String PPi network. The PPi network was performed to better understand BC behavior using the String Database and Cytoscape ${ }^{53,54}$. With the indicated cutoff of 0.9 , the final interactome network had 258 nodes conformed by 198 (86\%) proteins from the CS, PCA and PharmGKB/ CGI sets. Regarding nodes with the highest amount of genomic alterations showed previously in the OncoPrint, $65(89 \%)$ of them integrated this network (Fig. 5a). On the other hand, out of the 258 proteins that make up our String PPi network, 16 (6\%) proteins and 18 edges were part of the OncoPPi BC network ${ }^{55,56}$. The degree centrality made it possible to establish a significant correlation (Spearman test, $\mathrm{P}<0.05$ ) between our String PPi network and the OncoPPi BC network (Fig. 5b).

Considering degree centrality and consensus scores from our previous study ${ }^{29}$, there was enrichment among sub-networks (Fig. 5a,b). The degree centrality average in the whole network was 48.8, and out of the OncoPPi $\mathrm{BC}$ network was 124.4. Meanwhile, the average of consensus score of the whole network was 0.803 , and out of the OncoPPi BC network was 0.885 . As result, the second OncoOmics approach reveled 40 proteins with both the highest degree centrality and consensus score, as shown in Supplementary Table S19.

Protein expression analysis. The third OncoOmics approach was focused on proteins with considerable high and low expressions in BC. Figure 6a shows 43 proteins with significant high expression (Z-scores $\geq$ 2 ) and low expression (Z-scores $\leq-2)$ analyzed with the reverse-phase protein array (RPPA) and mass spectrometry, in a cohort of 994 individuals according to TCGA (Supplementary Table S20). On the other hand, the Human Protein Atlas (HPA) presented a map of the human tissue proteome based on tissue microarray-based immunohistochemistry. HPA has analyzed $202(88 \%)$ of the 230 proteins of our study, classifying the protein expression in high, medium, low and non-detected. As results, RAC1, GJB2, MED1, PIK3CA, PIK3R3, FGFR2, HCFC2, MAP2K4, NQO2 and RAC3 were proteins with high/medium expression in normal tissue, and low/ non-detected expression in BC tissue. Meanwhile, CDK2, CYP2D6, NCOR1, RRM1, FOXA1 and TOP2A were proteins with high/medium expression in $\mathrm{BC}$ tissue, and low/non-detected expression in normal tissue (F ig. $6 \mathrm{~b}$ and Supplementary Table S21 $)^{57,58}$. As result, the third OncoOmics approach revealed 60 proteins with significant altered expression levels as shown in Tables S20 and S21.

Breast cancer dependency map. The first analysis of the fourth OncoOmics approach consisted in identifying genes that are essential for breast cancer cell proliferation and survival performing systematic loss-of-function screens in a large number of well-annotated cell lines representing the tumor heterogeneity ${ }^{19-22}$. Figure 7a shows the distribution of dependency scores of 227 genes through DEMETER2, an analytical framework for analyzing genome-scale RNAi loss-of-function screens in 73 BC cell lines (Supplementary Table S22). Our results showed 563 dependencies with at least one score $\leq-1$ in $57(25 \%)$ essential genes. At the same time, Fig. 7a shows the distribution of dependency scores of 217 genes through CERES, an analytical framework for analyzing genome-scale CRISPR-Cas9 loss-of-function screens in 28 BC cell lines (Supplementary Table S23). Our results showed 310 dependencies with at least one score $\leq-1$ in 34 (16\%) essential genes. Figure $7 \mathrm{~b}$ shows the distribution of dependency scores of DEMETER2 and CERES per molecular subtype. The genome-scale RNAi loss-of-function screens detected 165 (29\%) dependencies in 19 Her2-enriched cell lines (ratio = 8.7), 110 (20\%) in 13 luminal A cell lines (8.5), 57 (10\%) in 7 luminal B cell lines (8.1), and 231 (41\%) in 34 basal-like cell lines (6.8), whereas the genome-scale CRISPR-Cas9 loss-of-function screens detected $85(27 \%)$ dependencies in 7 luminal A cell lines (ratio = 12.1), $176(15 \%)$ in 16 basal-like cell lines (11), and 49 (16\%) in 5 Her2-enriched cell lines (9.8). Figure $7 \mathrm{c}$ shows violin plots of dependencies per molecular subtype. DEMETER2 has detected a greatest number of substantial dependencies in basal-like, followed by Her2-enriched, luminal A and luminal B, whereas CERES has detected a greatest number of substantial dependencies in basal-like, followed by luminal A and Her2-enriched. Figure 7d shows a Venn diagram of 22 strongly selective genes, 26 common essential genes, and 5 strongly selective and common essential genes in breast and other cancer cell lines.

Patient-derived xenografts. The second analysis of the fourth OncoOmics approach consisted in identifying proteins with significant expression in PDXs. According to Woo et al., PDXs are in vivo models of human cancer that are useful for translational cancer research and therapy selection for individual patient. We analyzed the 66 strongly selective and common essential genes of BC cell lines using the Jackson Laboratory PDX resource $^{59}$. Figure 7 e shows 7 proteins with significant high expression (Z-score $\geq 2$ ) and 33 proteins with significant low expression (Z-scores $\leq-2)$ with its respective mice model ID. As result, the fourth OncoOmics approach revealed 38 proteins with significant expression in both BC cell lines and patient-derived xenografts (Supplementary Tables S22 and S23).

OncoOmics approaches to reveal essential genes in BC. After analyses of the four OncoOmics approaches (genomic alterations, String PPi network, protein expression and BC dependencies/patient-derived xenografts), we used a Venn diagram to integrate essential genes, termed OncoOmics BC essential genes. Consequently, we could observe 140 essential genes in at least one OncoOmics approach; of them, 92 were 


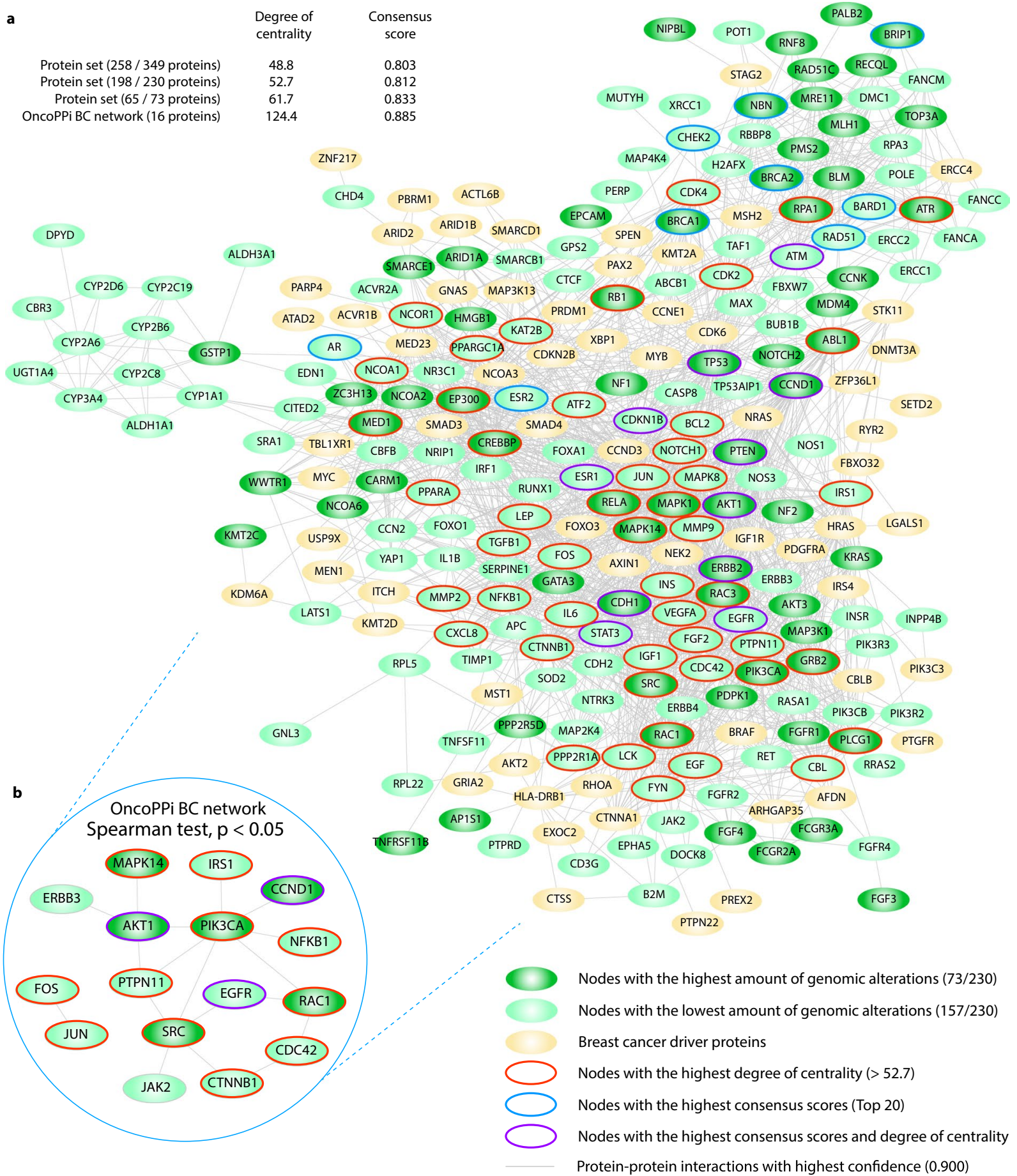

Figure 5. Protein-protein interactome network. (a) Network composed of BC driver genes and genes of our study (PCA gene set, consensus strategy gene set and PharmGKB gene set. (b) Significant correlation $(\mathrm{P}<0.05)$ of degree centrality and consensus score between the OncoPPi BC network and our String PPi network.

essential in one OncoOmics approach, 30 were essential in two OncoOmics approaches, 13 were essential in three OncoOmics approaches, and 5 were essential in all OncoOmics approaches as shown in Fig. 8a and Supplementary Table S24.

The 140 OncoOmics BC essential genes were conformed by oncogenes (21\%), tumor suppressor genes $(24 \%)$ and driver genes in other cancer types $(59 \%)^{60}$ (Fig. 8b). Additionally, some of these OncoOmics BC essential genes were involved in cancer immunotherapy ${ }^{61}$, kinome signaling ${ }^{62}$, cell cycle ${ }^{63}$, DNA repair ${ }^{64}$ and RNA-binding as shown in Fig. $8 \mathrm{c}$ and Supplementary Table S25 ${ }^{65}$.

Figure $8 \mathrm{~d}$ shows a circos plot detailing the correlation between 48 (34\%) OncoOmics BC essential genes and hallmarks of cancer. Suppression of growth was promoted by AKT1, CTNNB1, PTEN, RB1 and TP53; escaping 
a

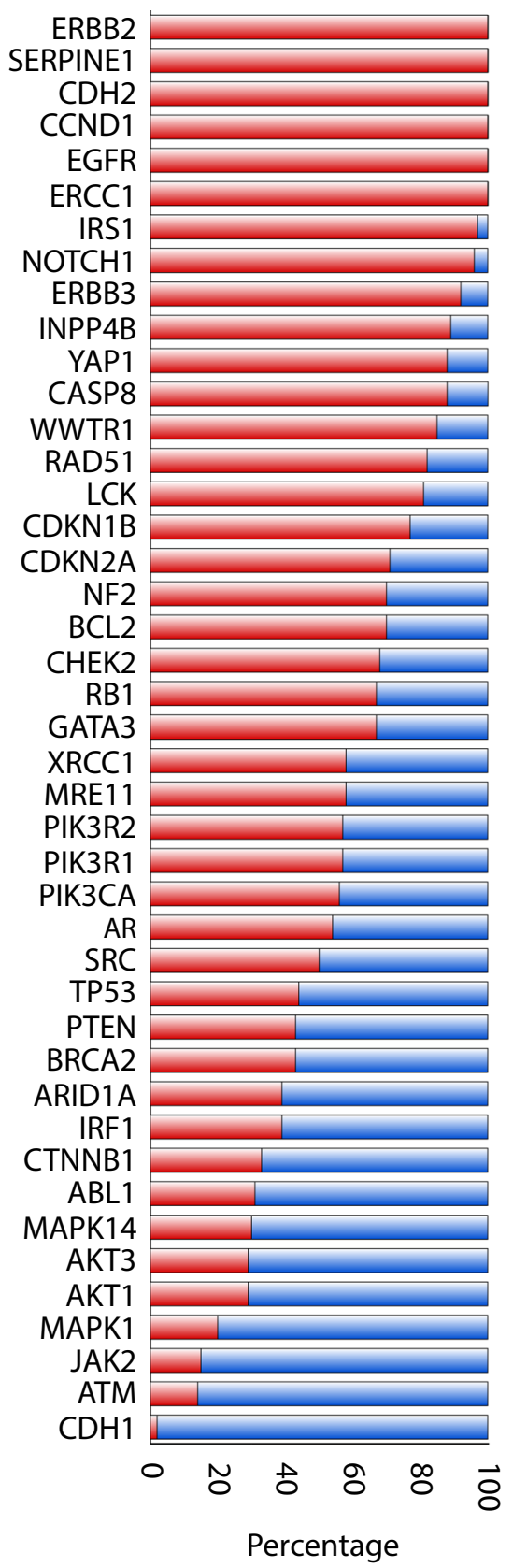

b

\section{2 of 230 proteins}

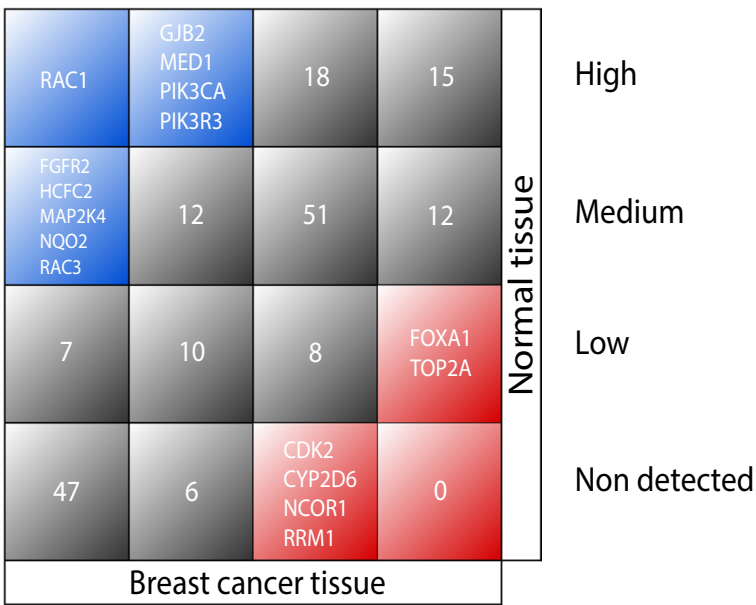

Protein low (Z-score $\leq-2)$

Protein high (Z-score $\geq 2)$

Figure 6. Protein expression analyses. (a) Proteins $(n=43)$ with alterations in the expression levels. Low expression proteins with Z-score $\leq-2$ and high expression proteins with Z-score $\geq 2$ according to TCGA. (b) Comparison of protein expression levels $(n=202)$ by immunohistochemistry between $B C$ tissue and normal tissue according to The Human Protein Atlas.

immune response to cancer was promoted by CTNNB1, EGFR and RAC1; cell replicative immortality was promoted by CTNNB1, KRAS and NOTCH1; tumor promoting inflammation was promoted by KRAS; metastasis was promoted by $A B L 1, C T N N B 1, E G F R, K R A S, R A C 1$ and $R B 1$; angiogenesis was promoted by $A B L 1, C T N N B 1$, $E G F R, K R A S$, NOTCH1 and $R A C 1$; genome instability was promoted by $A B L 1$ and $R B 1$; escaping programmed cell death was promoted by $A K T 1, C T N N B 1, E G F R$, NOTCH1; change of cellular energetics was promoted by $A B L 1, A K T 1, C T N N B 1, E G F R, K R A S, N O T C H 1, P T E N, R B 1$ and TP53; finally, proliferative signaling was promoted by ABL1, AKT1, CTNNB1, EGFR, KRAS, NOTCH and RAC1 (Supplementary Table S26).

Enrichment map of the OncoOmics BC essential genes. Figure 8e shows the enrichment map of the 140 OncoOmics BC essential genes. g:Profiler searches for a collection of genes representing GO terms, pathways and disease phenotypes ${ }^{66}$. The most significant GO: biological processes with a FDR $<0.001$ was positive 
a

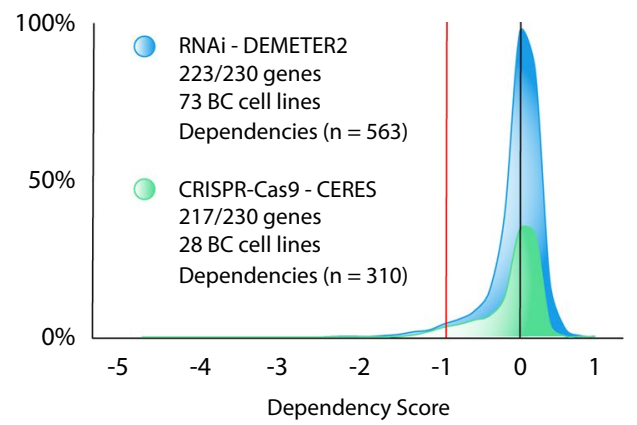

c

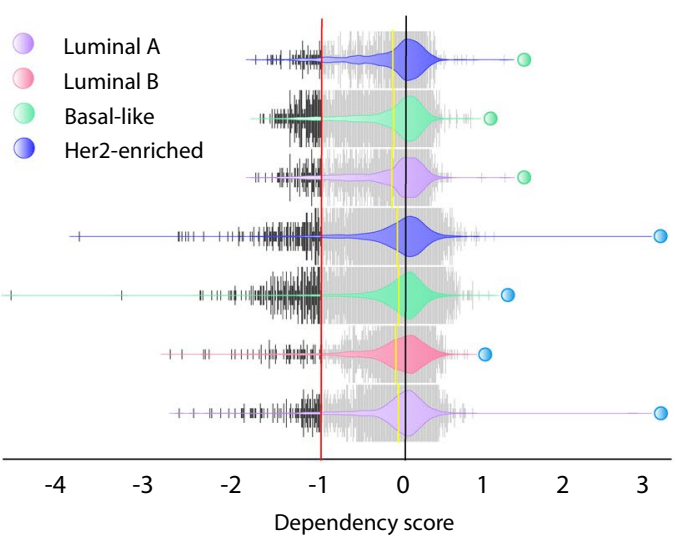

b

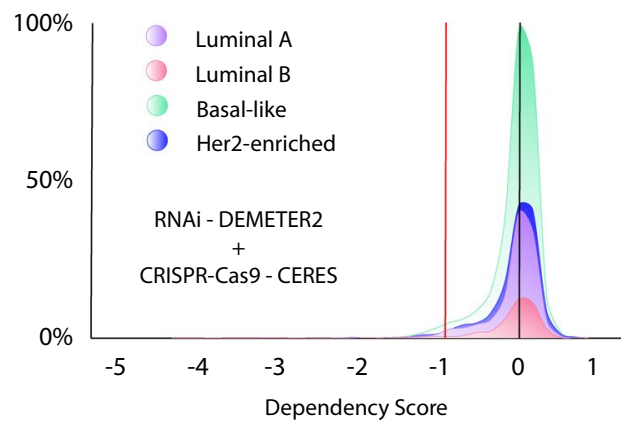

d

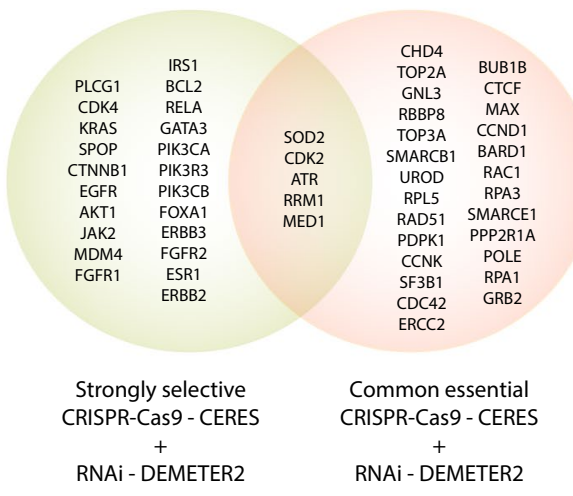

Dependent cell lines: CRISPR-Cas9 $(n=558)$, RNAi $(n=711)$

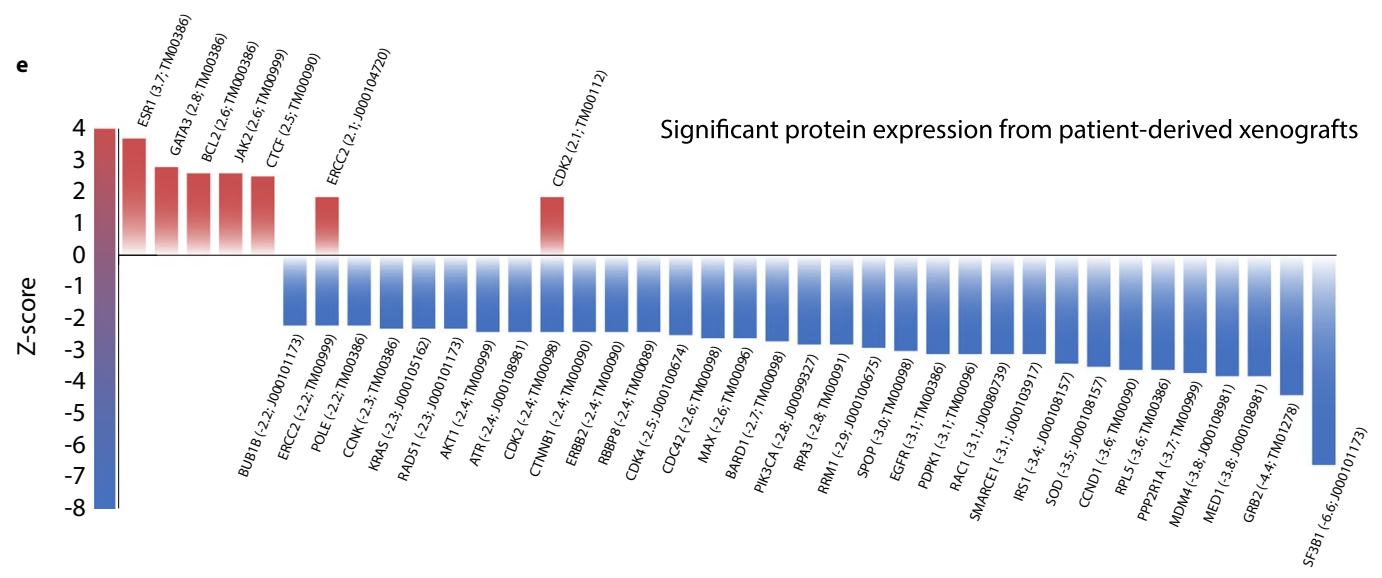

Figure 7. BC dependency maps in cell lines and patient-derived xenografts. (a) Dependency score of gene sets using RNAi DIMETER2 and CRISPR-Cas9 CERES algorithms in BC cell lines. (b) Dependency score of BC gene sets per molecular subtypes. (c) Violin plots of dependencies per molecular subtypes. All substantial dependencies $<-1$ are in black. (d) Venn diagram of strongly selective and common essential genes in all cancer cell lines. (e) Significant protein expression from patient-derived xenografts.

regulation of macromolecule metabolic process (Supplementary Table S27); the most significant GO: molecular function was phosphatidylinositol 3-kinase activity (Supplementary Table S28); the most significant Reactome pathway was generic transcriptor pathway (Supplementary Table S29) ${ }^{67}$; additionally, the most relevant disease, according the Human Phenotype Ontology, was breast carcinoma (Supplementary Table S30 ${ }^{68}$. Subsequently, g:Profiler annotations were analyzed with the EnrichmentMap software and visualized using Cytoscape, in order to generate network interactions of the most relevant GO: biological processes (Supplementary Fig. S2) and Reactome pathways (Fig. 9) related to immune system, tyrosine kinase, cell cycle and DNA repair pathways ${ }^{54,66}$.

Clinical trials. Figure 10 and Supplementary Table S31 details the current status of clinical trials regarding OncoOmics BC essential proteins, according to the Open Targets Platform ${ }^{69}$. There are 98 drugs that are being analyzed in 2,904 clinical trials in 28 of 140 OncoOmics BC essential proteins (Fig. 10a). The top 10 drugs with the highest number of clinical trials in process or completed were paclitaxel (370), trastuzumab (315), docetaxel 
a
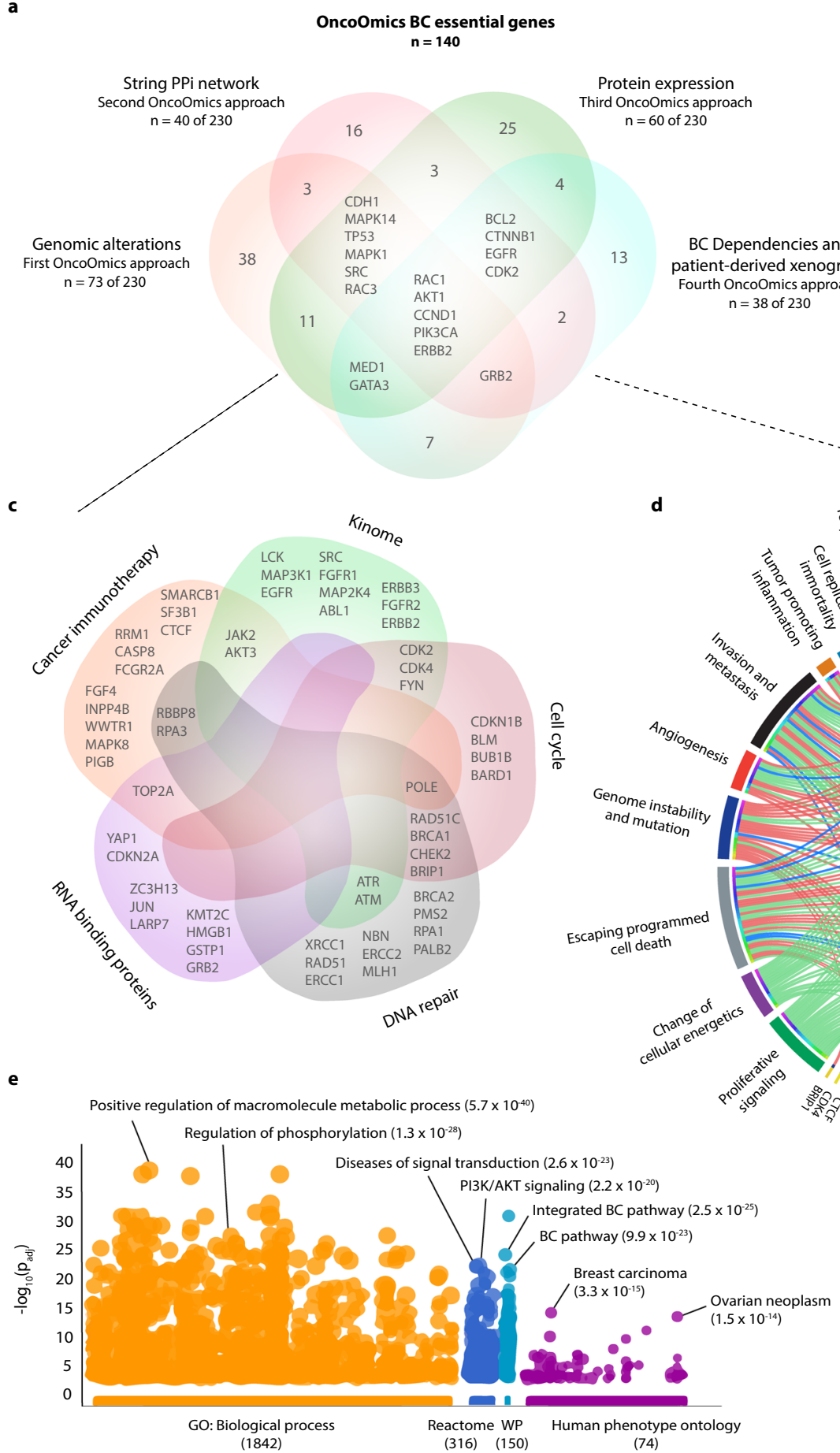

b

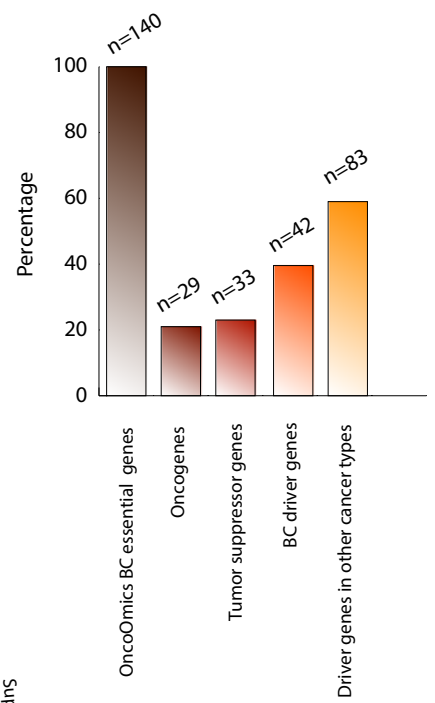

Figure 8. OncoOmics BC essential genes. (a) Venn diagram of the most essential genes per genomics approach (genomic alterations, String PPi network, protein expression, and BC dependencies/patient-derived xenografts). (b) Percentage of oncogenes, tumor suppressor genes and driver genes in other cancer types. (c) Venn diagram of the most essential genes related to cancer immunotherapy, kinome signaling, cell cycle, DNA repair and RNA-binding proteins. (d) Circos plot of genes with hallmarks of cancer. (e) Most significant g:Profiler features of the OncoOmics BC essential genes according to GO: biological processes, Reactome pathways, WikiPathways and the human phenotype ontology.

(262), doxorubicin (204), gemcitabine (196), lapatinib (152), tamoxifen (131), fulvestrant (129), bevacizumab (120) and neratinib (110). Regarding drugs, $94 \%$ were antagonists, $79 \%$ were small molecules, and $35 \%$ were protein kinases as shown in Fig. 10b-d, respectively. Additionally, drugs with the highest number of clinical trials in phases 3 and 4 were paclitaxel (111), docetaxel (105), trastuzumab (80), tamoxifen (69) and doxorubicin (60) as shown in a Sankey plot detailed in Fig. 10e. 


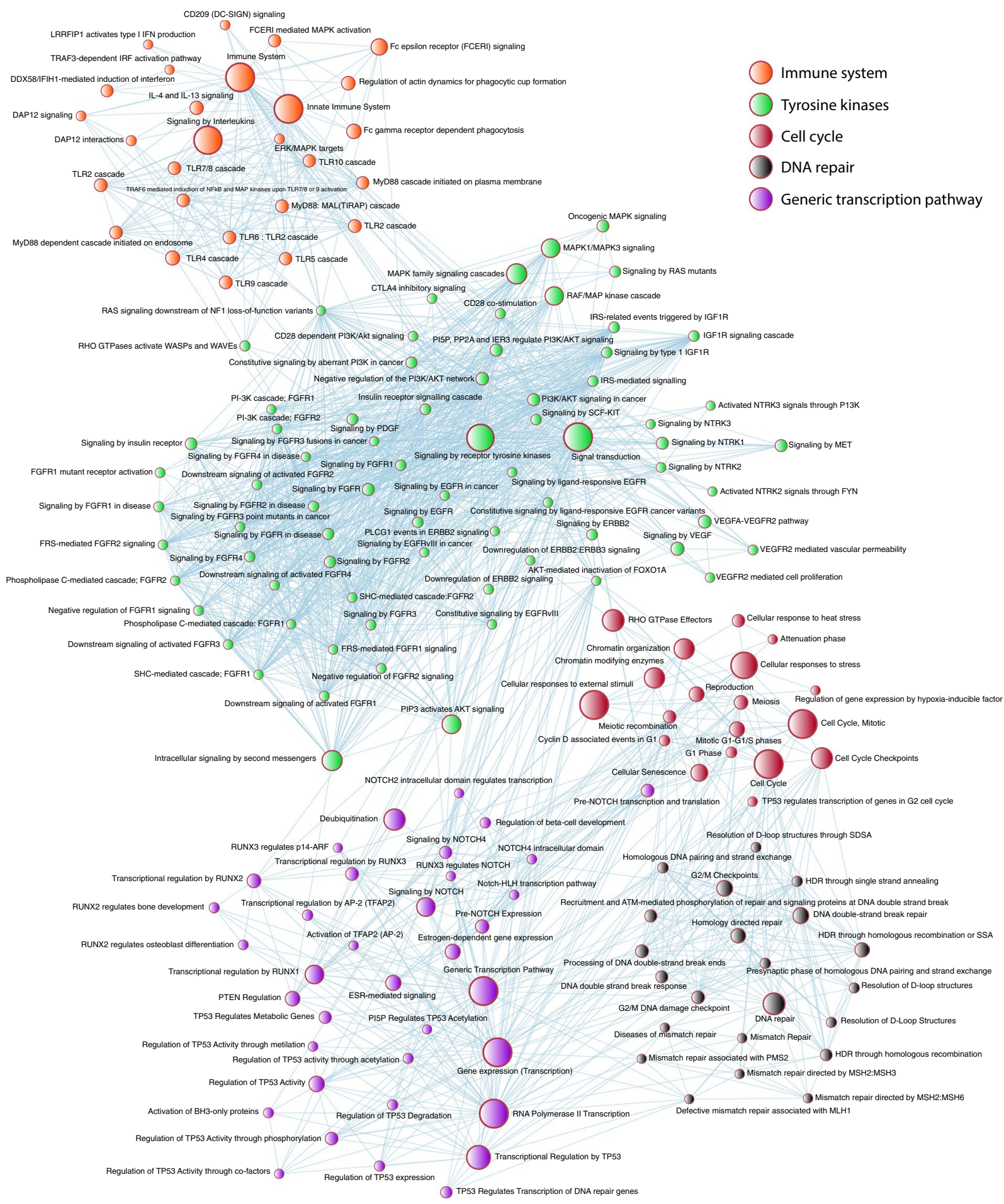

Figure 9. Pathway enrichment analysis of the OncoOmics BC essential genes using g:Profiler and

EnrichmentMap. Most significant Reactome pathways related to immune system, kinome signaling, cell cycle, DNA repair and genetic transcription.

Precision medicine. Precision oncology focuses on matching the most effective and safe treatment based on the 'omics' profile of each individual or population ${ }^{70,71}$. However, the identification of driver mutational events remains the biggest challenge ${ }^{72}$. There are some consortiums and studies that have robustly identified variants associated with BC. Tamborero et al. detailed a compendium of 62 somatic and 398 germline validated oncogenic mutations in 14 OncoOmics BC essential genes (Supplementary Table S32) ${ }^{38}$. Huang et al. identified 87 pathogenic germline variants in 22 OncoOmics BC essential genes ${ }^{73}$ (Supplementary Table S33). Long et al. ${ }^{74,75}$, Cai et al. ${ }^{76}$, Michailidou et al. ${ }^{77}$, and the Breast Cancer Association Consortium performed genome-wide association 
a

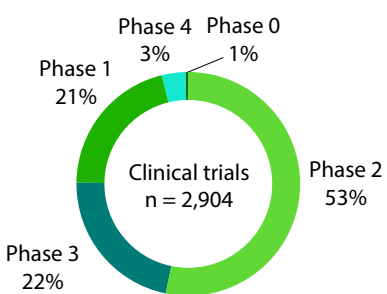

b

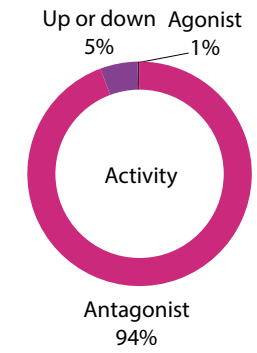

C

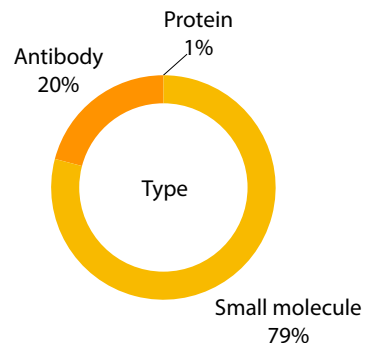

d

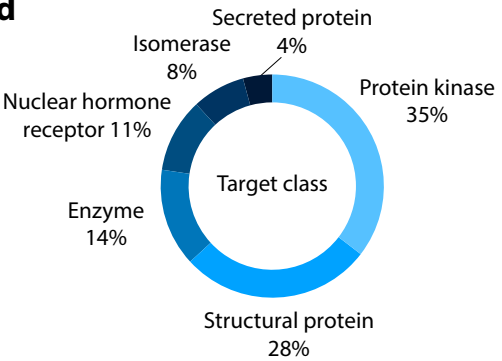

e

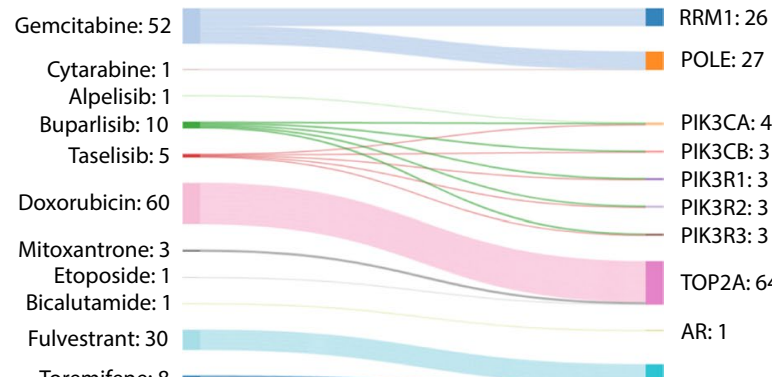

ESR1: 107

Tamoxifen: 69

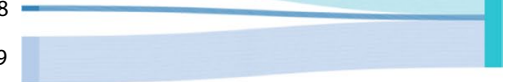

ESR: 107

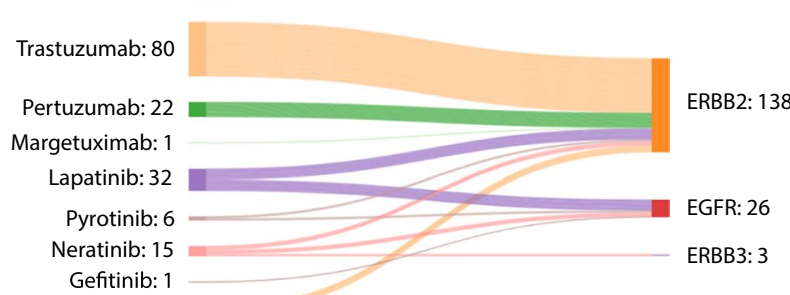
Trastuzumab emtansine: 20

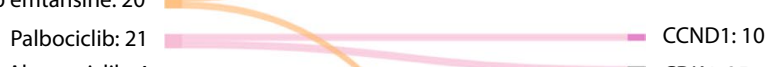

Abemaciclib: $4 \longrightarrow$ CDK4: 25

Ribociclib: 10

Bevacizumab: 25

Vinorelbine: 17

Paclitaxel: 111

Eribulin: 14

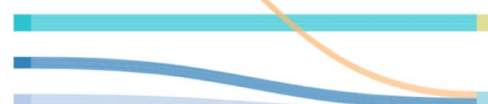

VEGFA: 25

Docetaxel: 105

Ixabepirone: 8

Vinflunine: 3

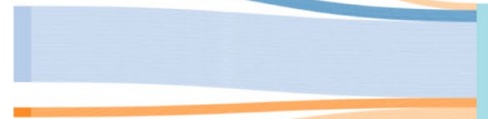

TUBB1: 268

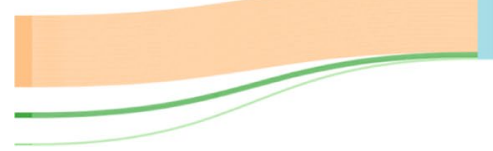

736 clinical trials in phases 3 and 4

Figure 10. Current status of clinical trials in the OncoOmics BC essential proteins. (a) Clinical trials per phase. (b) Clinical trials per activity. (c) Clinical trials per type. (d) Clinical trials per target class. (e) Correlation of drugs with proteins in advanced stages of clinical trials (3 and 4) using a Sankey plot.

studies identifying 172 germline variations related to BC development (Supplementary Table S34). The Precision Medicine Knowledgebase (PreMedKB) detailed a compendium of 2791 germline variants in 7 OncoOmics BC essential genes (Supplementary Table S35) ${ }^{71}$. PharmGKB enriched clinical guidelines with 59 well-known clinical annotations related to 29 OncoOmics BC essential genes (Supplementary Table S36) ${ }^{42,78,79}$. Finally, the Pan-Cancer Analysis of Whole Genomes (PCAWG) Consortium identified 19 non-coding somatic mutations and 17 coding somatic mutations in BC (Supplementary Table S37) ${ }^{6}$.

Regarding the Ensembl Variant Effect Predictor ${ }^{80}, 1,102$ of 3,565 variants were processed, being $24 \%$ intron variants, $16 \%$ missense variants, $15 \%$ downstream gene variants, $10 \%$ stop gained, $7 \%$ upstream gene variants, $7 \%$ NMD transcript variants, $4 \%$ splice region variants, $4 \% 3^{\prime}$ untranstaled region variants, and $2 \%$ splice acceptor variants (Supplementary Table S38).

Consequently, based on the aforementioned somatic and germline oncogenic variants, the Cancer Genome Interpreter and PreMedKB platforms provided a comprehensive in silico list of biological therapy drugs aimed to improve precision medicine in breast cancer (Fig. 11, Tables S35 and S39). 


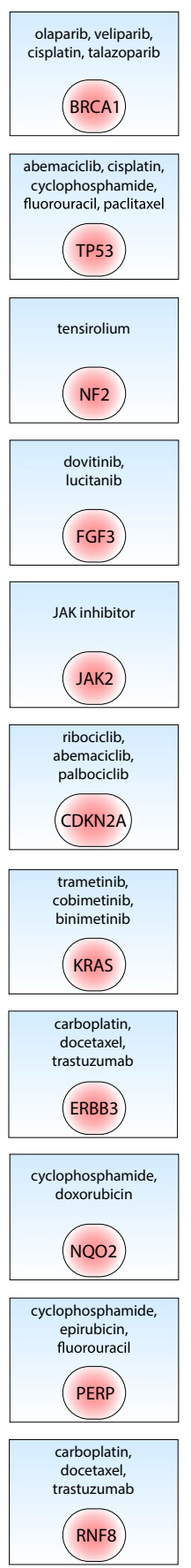

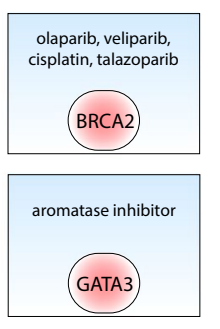
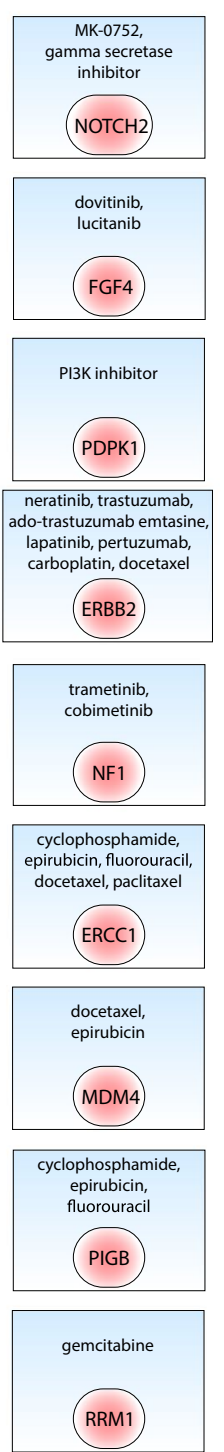
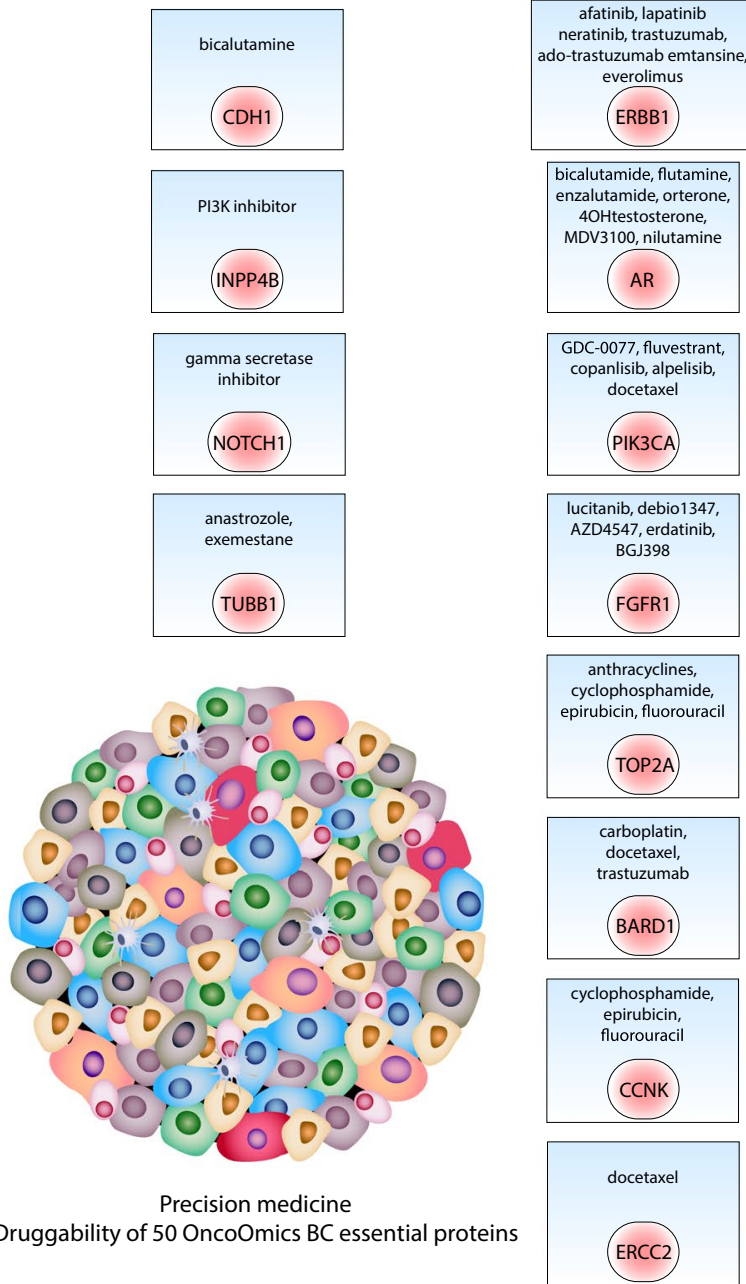

Druggability of 50 OncoOmics BC essential proteins
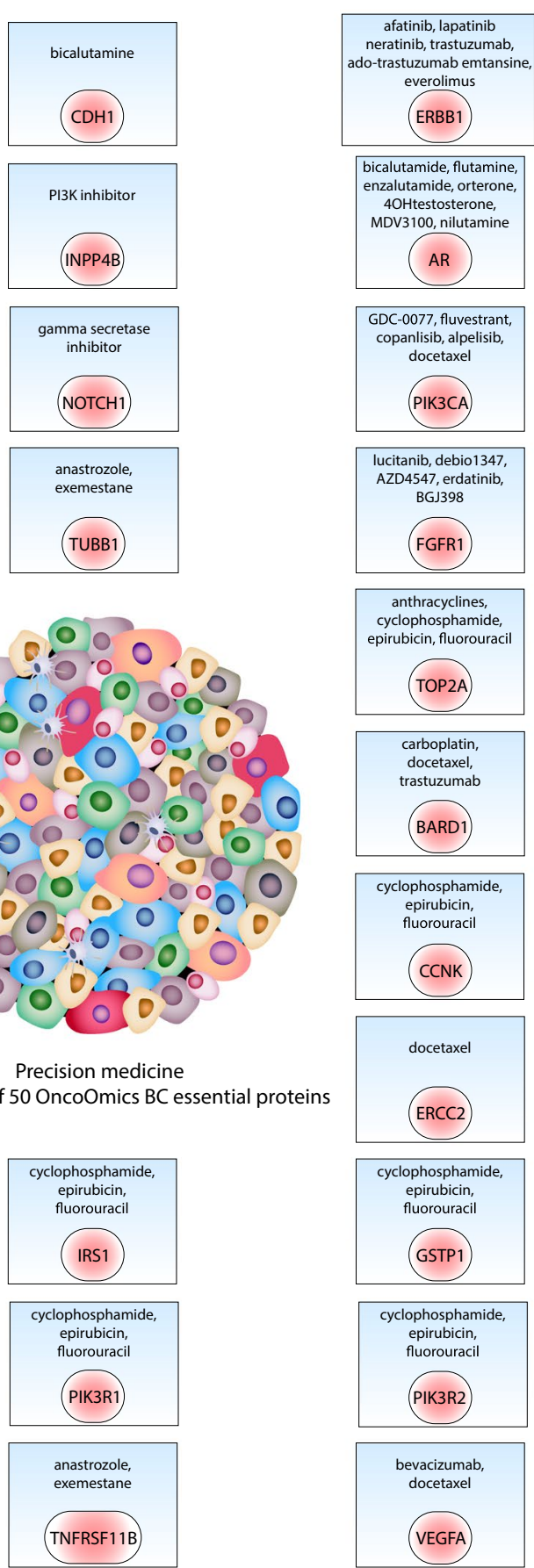

lucitanib, debio1347, AZD4547, erdatini

BGJ398

FGFR1

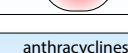

cyclophosphamide,

(TOP
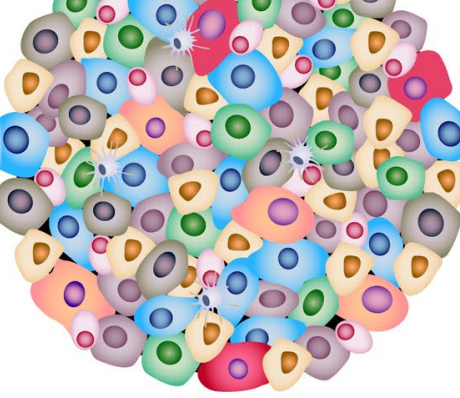

Precision medicine
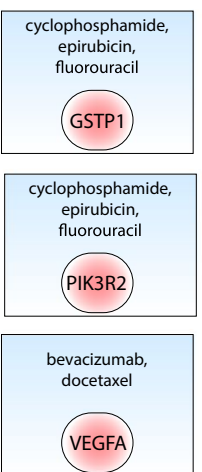
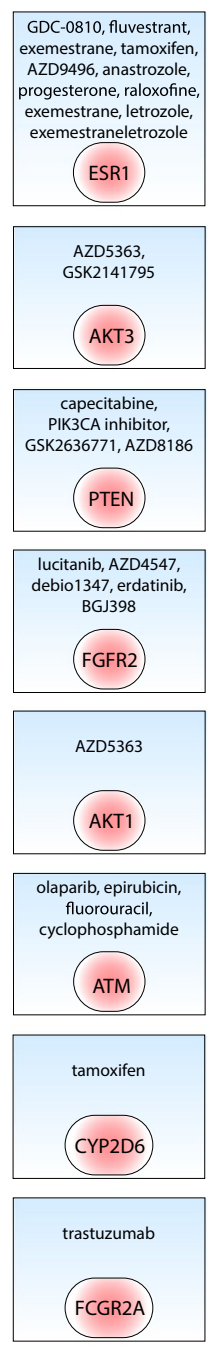

trastuzumab
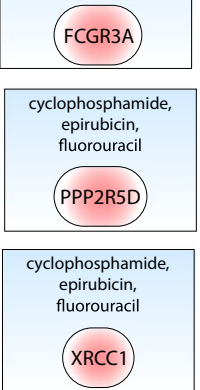

Figure 11. Precision medicine. Interaction between drugs and 50 OncoOmics $\mathrm{BC}$ essential proteins.

\section{Discussion}

In this study we reveal essential genes in breast cancer through an OncoOmics strategy that analyzes genomic alterations, PPi networking, protein expression, dependency maps and patient-derived xenografts in three gene sets. The first gene set was taken from our previous study where we developed a Consensus Strategy that was proved to be highly efficient in the recognition of $\mathrm{BC}$ pathogenic genes ${ }^{29,41}$. The second gene set was taken from several studies of PCA, which provides a panoramic view of the oncogenic processes that contributes to BC pathogenesis ${ }^{3,13,31-37}$. The third gene set was taken from the CGI and PharmGKB. On the one hand, the CGI flags genomic biomarkers of drug response with different levels of clinical relevance ${ }^{38}$. On the other hand, PharmGKB collects clinical annotations applied in BC patients and taken from the NCCN, ESMO, CPNDS, DPWG and CPIC guidelines $^{43-46}$. Finally, the compendium of these 230 genes was analyzed through four different OncoOmics approaches. 
The first OncoOmics approach consisted in the analysis of genomic alterations using the PCA data ${ }^{47,48}$. The frequency mean of genomic alterations in the CS (1.2) and PCA (1.3) gene sets were significantly higher than both the non-cancer genes (0.4) and the well-known BC driver genes (0.8), with a significant Bonferroni correction of $\mathrm{P}<0.001$. This means that the analyzed set of genes might be strongly associated with BC (Fig. 1a).

The most common genomic alterations in a cohort of 994 individuals were mRNA up-regulation, CNV amplification and missense mutations. Regarding molecular subtypes, basal-like showed the highest amount of genomic alterations. PIK3CA was the most altered gene in luminal A, CCND1 in luminal B, TP53 in basal-like and normal-like, and ERBB2 in Her2-enriched (Fig. 2a). A multiple comparison through Bonferroni correction found significant differences $(\mathrm{P}<0.05)$ of $\mathrm{CNV}$ amplifications, $\mathrm{CNV}$ deep deletions, mRNA up-regulations, and mRNA down-regulations among molecular subtypes (Figs. 2c-f). Regarding tumor stages, T2 showed the highest amount of genomic alterations. PIK3CA was the most altered gene in T1, TP53 in T2 and T3, and ERBB2 in T4 (Fig. 2g). Bonferroni correction found significant differences $(\mathrm{P}<0.05)$ in punctual mutations, CNV amplifications, CNV deep deletions, mRNA up-regulations, and mRNA down-regulations among tumor stages (Fig. 2h-1). Lastly, the first OncoOmics approach revealed that 73 essential genes presented frequencies of alteration higher than the average (Fig. 3a) $)^{3,13,31-37}$.

Subsequently, the enrichment analysis of signaling pathways was carried on taking into account all genomic alterations in the 230 genes using David Bioinformatics Resource and $\mathrm{KEGG}^{49,52}$. Pathways with the highest amount of genomic alterations per molecular subtype were Jak-STAT in luminal A, Wnt in luminal B, p53 in basal-like, ERBB in Her2-enriched and Hippo in normal-like. Bonferroni correction showed significant differences $(\mathrm{P}<0.05)$ among several subtypes as shown in Fig. $4 \mathrm{~b}$. On the other hand, pathways with the highest amount of genomic alterations per tumor stage were Wnt in T1, T2 and T3, and thyroid hormone in T4. Bonferroni correction showed significant differences $(\mathrm{P}<0.05)$ comparing $\mathrm{T} 1$ with $\mathrm{T} 2$ and $\mathrm{T} 4$ as shown in Fig. 4d.

Regarding previously mentioned signaling pathways, Jak-STAT is involved in inflammatory response, stem cell maintenance, and hematopoiesis ${ }^{81}$. The Wnt signaling pathway actively functions in embryonic development and helps in homeostasis in mature tissues by regulating cell survival, migration, proliferation, and polarity ${ }^{82}$. The p53 signaling pathway plays an essential role into inhibition of growth, programmed cell death, cell migration and angiogenesis ${ }^{83}$. The ERBB pathway mediates signal transduction events that control cell survival, migration and proliferation in $\mathrm{BC}^{84}$. The Hippo pathway plays important roles in tumor suppression and immune response. However, alterations in this pathway are involved in the $\mathrm{BC}$ tumorigenesis and metastasis ${ }^{85}$. Lastly, the thyroid hormone pathway plays an important role as regulator of growth and metabolism. Nevertheless, dysfunction of the T3 hormone promotes cancer progression in mammary epithelial cells ${ }^{86}$.

The second OncoOmics approach was focused on proteins with the highest degree centrality and consensus score in the String PPi network. In accordance with Li et al. and Ivanov et al. ${ }^{56,87}$, PPi with therapeutic significance can be revealed by the integration of cancer proteins into networks. PPi regulate essential oncogenic signals to cell proliferation and survival, and thus, represents potential targets for drug development and drug discovery. Regarding our networking analysis, the final interaction network consisted in 258 nodes with a degree centrality average of 48.8 and a consensus score average of $0.803^{29}$; the sub-network integrated by 198 of 230 nodes had 52.7 of degree centrality and 0.812 of consensus scoring; finally, the sub-network integrated by 65 of 73 proteins with the highest amount of genomic alterations had 61.7 of degree centrality and 0.833 of consensus score. Hence, a sub-network of nodes with the highest amount of genomic alterations presented a highest degree centrality and consensus score, suggesting that there is strong correlation between these proteins and BC. Additionally, the oncogenomics validation showed a substantial correlation between our String PPi network (Fig. 5a) and the OncoPPi BC network (Fig. 5b), identifying 16 nodes strongly associated with $\mathrm{BC}^{29}$. The second OncoOmics approach revealed 40 essential proteins with the highest degree centrality and consensus scoring.

The third OncoOmics approach was focused on proteins with significant high and low expression in BC proteome. More than 500 proteins have been identified as strongly involved in oncogenesis. Loss of expression, overexpression or expression of dysfunctional proteins contribute to uncontrolled tumor growth, causing chromosomal rearrangements, gene amplification and ungoverned methylation ${ }^{88}$. Regarding our 230 proteins, 43 showed significant high (Z-scores $\geq 2$ ) and low (Z-scores $\leq-2$ ) expression according to TCGA ${ }^{89}$ (Fig. 6a); and 16 proteins showed opposite expression between healthy and affected tissues after microarray-based immunohis-

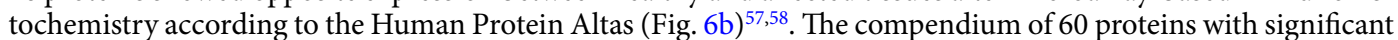
high and low expressions made up the third OncoOmics approach.

The fourth OncoOmics approach was related to the BC dependency map in cell lines and patient-derived xenografts. According to Tsherniak et al., mutations that trigger the growth of cancer cells also confer specific vulnerabilities that normal cells lack, and these dependencies are compelling therapeutic targets ${ }^{19}$. The cancer dependency map identifies essential genes in proliferation and survival of well-annotated cell lines through systematic loss-of-function screens ${ }^{19-22}$. On the one hand, DETEMER2 analyzed the genome-scale RNAi loss-of-function screens, and on the other hand, CERES analyzed the genome-scale CRISPR-Cas9 loss-of-function screens as shown in Fig. 7a. In addition to the loss-of-function screens in a large number of well-annotated BC cell lines, the patient-derived xenografts are in vivo models of human tumors engrafted in a mouse host and emerging as a powerful tool for understanding tumor hallmarks and predicting drug effi$\operatorname{cacy}^{90}$. Consequently, we validated the genomic expression of the strongly selective and common essential genes (dependencies in BC cell lines) in breast tumors from PDXs provided by the Jackson Laboratory ${ }^{59}$. The fourth OncoOmics approach was made up of 38 essential proteins in BC (Fig. 7e).

Subsequently, the compendium of essential genes per approach reveals the 140 OncoOmics BC essential genes (Fig. 8a). RAC1, AKT1, CCND1, PIK3CA and ERBB2 were essential genes in all the OncoOmics approaches. $C D H 1, M A P K 14$, TP53, MAPK1, SRC and RAC3 showed genomic alterations, highest degree centrality and consensus scores in the String PPi network, and significant protein expression. GRB2 showed genomic alterations, 
highest degree centrality and consensus scores in the String PPi network, and substantial relevance in BC cell lines and PDXs. MED1 and GATA3 showed genomic alterations, significant protein expression, and considerable relevance in BC cell lines and PDXs. Lastly, BCL2, CTNNB1, EGFR and CDK2 showed significant protein expression, highest degree centrality and consensus scores in the String PPi network, and substantial relevance in BC cell lines and PDXs.

Relevant studies worldwide have identified OncoOmics BC essential genes. For instance, genome-wide association studies performed by the Breast Cancer Association Consortium showed that BRCA2, CHEK2, ESR1, FGFR2, MDM4 and PIK3R3 carry germline variants associated with BC development ${ }^{74-77}$. According to Bailey et al., identifying molecular cancer drivers is critical for precision oncology ${ }^{32}$. Their final consensus list was conformed by $29 \mathrm{BC}$ driver genes, of them, 22 were OncoOmics BC essential genes (AKT1, ARID1A, BRCA1, CASP8, CDH1, CDKN1B, CTCF, ERBB2, FOXA1, GATA3, KMT2C, KRAS, MAP2K4, MAP3K1, NCOR1, NF1, PIK3CA, PIK3R1, PTEN, RB1, SF3B1 and TP53). According to Gonzalez-Perez et al., the IntOGen-mutation platform summarizes somatic mutations involved in tumorigenesis ${ }^{91}$. Their final consensus list was conformed by 99 mutational BC driver genes, of them, 34 were identified by the OncoOmics strategy (TP53, PIK3CA, KMT2C, GATA3, CDH1, MAP3K1, ESR1, PTEN, AKT1, NCOR1, ARID1A, MAP2K4, FOXA1, NF1, ERBB2, RB1, SF3B1, ERBB3, CTCF, PIK3R1, ATM, FGFR2, BRCA1, CASP8, CREBBP, BRCA2, CDKN2A, KRAS, CDKN1B, NOTCH2, MAX, $M D M 4, E G F R$ and JAK2). Finally, the PCAWG Consortium of the International Cancer Genome Consortium (ICGC) and The Cancer Genome Atlas reported an integrative analysis of 2,658 whole-cancer genomes across 38 tumor types $^{92}$. Regarding breast cancer, PCAWG identified 27 mutational BC driver genes, of them, 15 were OncoOmics BC essential genes (TP53, PIK3CA, MAP3K1, KMT2C, NOTCH2, SF3B1, PTEN, ARID1A, MAP2K4, $A K T 1, C T C F, F O X A 1, R B 1, C D K N 2 A$ and $A T M)$.

According to Reimand et al., g:Profiler lets us know the enrichment map of the 140 OncoOmics BC essential genes ${ }^{66}$. The most significant GO: biological process was the positive regulation of macromolecule metabolic process, the GO: molecular function was phosphatidylinositol 3-kinase activity, the Reactome pathway was generic transcriptor pathway, and the most significant Human Phenotype Ontology term was breast carcinoma ${ }^{68}$. Subsequently, the most relevant network interactions of the GO: biological process and the Reactome pathways were related to immune system, tyrosine kinase, cell cycle and DNA repair terms (Figs. 9 and S2) ${ }^{54,66}$.

There is currently great enthusiasm about immunotherapeutic strategies to treat $\mathrm{BC}^{93}$. The first approval of an immune checkpoint blockade agent for treatment of BC came in March 2019 when the anti-PD-L1 antibody atezolizumab was approved to be used with nab-paclitaxel in triple-negative BC patients ${ }^{94,95}$. 16 OncoOmics BC essential genes were associated with immunotherapy ${ }^{61,96}$ as shown in Fig. $8 \mathrm{C}$. Kinases have been recognized as therapeutic targets due to their druggability and play a critical role in cell migration, differentiation, growth and survival $^{97} .15$ OncoOmics BC essential genes were kinomes ${ }^{62}$. Cell cycle comprises a series of events that drive cell division and DNA replication ${ }^{98} .12$ OncoOmics BC essential genes were involved in cell cycle ${ }^{63}$. DNA repair signaling pathways work in concert to correct DNA lesions and maintain genome stability. Nevertheless, a defective DNA repair machinery causes $\mathrm{BC}$ development and progression ${ }^{99} .17$ OncoOmics BC essential genes were involved in DNA repair ${ }^{64}$. RBPs are key players in post-transcriptional events and are emerging as critical modulators in $\mathrm{BC}^{100-102}$. Bioinformatics profiling of tumors have revealed the landscape of alterations in RBPs across cancer types ${ }^{103-106}$. Lastly, 10 OncoOmics BC essential genes were RBPs ${ }^{65}$.

Regarding clinical trials reported on the OncoOmics BC essential proteins, the Open Targets Platform is an available resource for the integration of genomics and chemical data to aid systematic drug target identification and prioritization ${ }^{69}$. There are 98 drugs that are being analyzed in 2,904 clinical trials in 28 of 140 OncoOmics $\mathrm{BC}$ essential proteins. Additionally, there are 30 drugs involved in 736 clinical trials in phases 3 and 4 . The top five drugs with the highest number of clinical trials in process or completed are paclitaxel (111), docetaxel (105), trastuzumab (80), tamoxifen (69), and doxorubicin $(60)^{69}$ (Fig. 10e).

Tumor-related genomic alterations predict tumor prognosis, drug response, and toxicity ${ }^{107}$. Precision medicine provides patients with the most appropriate diagnostics and targeted therapies based on the 'omics' profile and other predictive and prognostic tests ${ }^{108}$. Therefore, precision medicine aims to deliver the right medicine to the right patient at the right dose at the right time, minimizing adverse effects and maximizing drug efficacy ${ }^{109,110}$. Figure 11 shows comprehensive interactions between directed biological drugs and 50 OncoOmics BC essential proteins aimed to improve precision medicine in breast cancer.

In conclusion, since $\mathrm{BC}$ is a complex and heterogeneous disease, the study of different OncoOmics approaches is an effective way to reveal essential genes to better understand the molecular landscape of processes behind oncogenesis, and to develop better therapeutic treatments focused on pharmacogenomics and precision medicine.

\section{Methods}

OncoPrint of genomic alterations according to the Pan-Cancer Atlas. PCA has reported the clinical data of 1084 individuals with BC and it can be visualized in the Genomic Data Commons of the National Cancer Institute (https://gdc.cancer.gov/) and in the cBioPortal (http://www.cbioportal.org/4 ${ }^{47,48}$. The clinical annotations were age, pTNM classification, tumor type, tumor stage and race/ethnicity.

Additionally, PCA has reported genomic alterations (mRNA up-regulation, mRNA down-regulation, CNV amplification, CVN deep deletion, putative driver mutations and fusion gene) of 994 individuals. Putative mutations were analyzed through exome sequencing, CNVs through the Genomic Identification of Significant Targets in Cancer (GISTIC 2.0) ${ }^{111,112}$, and mRNA expression through RNA Seq V2. We analyzed five gene sets in order to compare the frequency mean of genomic alterations among them. The first gene set $(n=177)$ was integrated by the non-cancer genes ${ }^{113}$. We calculated the OncoScore of non-cancer genes, taking out all genes from our study. The second gene set $(n=119)$ was the BC driver genes, according to The Network of Cancer Genes ${ }^{60}$. The third gene set $(n=84)$ was taken from our previous study where we developed a Consensus Strategy of prioritized 
genes related to $\mathrm{BC}$ pathogenesis ${ }^{29}$. The fourth gene set $(n=85)$ was made up of genes associated with $\mathrm{BC}$ development, according to several PCA studies ${ }^{31,32,114}$. Finally, the fifth gene set $(n=91)$ consisted of BC biomarkers and druggable enzymes taken from PharmGKB and the CGI (Supplementary Table S2) $38,39,42$.

The OncoOmics approaches were performed in 230 genes conformed by the CS, PCA and PharmGKB/ CGI gene sets. We calculated the percentage and ratio of genomic alterations per intrinsic molecular subtype and tumor stage, and then we established a ranking of genes with the highest amount of genomic alterations (OncoPrint). The OncoPrint conformed the first OncoOmics approach.

Pathway enrichment analysis. The enrichment analysis of signaling pathways was performed using David Bioinformatics Resource to obtain integrated information from KEGG ${ }^{49-52}$. It was carried on in the 230 genes, taking into account terms with a significant FDR $<0.01$. After that, genomic alterations that comprise each signaling pathway were analyzed, taking into account the molecular subtype and tumor stage of individuals from PCA. Circos plots and violin plots were designed to visualize all data. Lastly, in order to compare the ratio of genomic alterations among subtypes and tumor stages, normalization was carried out dividing the number of genomic alterations by the number of individuals per subtype and tumor stage. Regarding molecular subtypes, 499 individuals were luminal A, 197 were luminal B, 171 were basal-like, 78 were Her2-enriched and 36 were normal-like, and regarding tumor stage, 255 were T1, 586 were T2, 113 were T3, and 103 were T4.

Protein-protein interactome network. The PPi network with a highest confidence cutoff of 0.9 and zero node addition was created using the String Database, which takes into account predicted and known interactions ${ }^{53}$. The confidence scoring is the approximate probability that a predicted link exists between two enzymes in the same metabolic map, whereas the degree centrality of a node means the number of edges the node has to other nodes in a network. The centrality indexes calculation and network visualization were analyzed through the Cytoscape software ${ }^{54}$. Proteins with the highest degree centrality, consensus score and sub-networks were differentiated by colors in the PPi network. On the other hand, OncoPPi (http://oncoppi.emory.edu/) reports the development of a cancer-focused PPi network, identifying more than 260 high-confidence cancer-associated $\mathrm{PPi}^{55,56}$. In addition, the OncoPPi BC network consisted of 16 proteins and $18 \mathrm{PPi}$ experimentally analyzed in $\mathrm{BC}$ cell lines $^{55,56}$. The correlation of the degree centrality by means of Spearman P-value test between our String PPi network and the OncoPPi BC network allowed for the validation of all the high-confidence BC-focused PPi analyzed in cell lines ${ }^{29}$. Lastly, proteins with the highest degree centrality and consensus scoring made up the second OncoOmics approach.

Protein expression analysis. TCGA has reported the protein expression data of 994 individuals with BC through RPPA and mass spectrometry by the Clinical Proteomic Tumor Analysis Consortium (CPTAC), and it can be visualized in the $\mathrm{cBioPortal}^{47,48}$. We analyzed the protein expression of 230 protein where Z-scores $\geq 2$ mean a significant high protein expression and Z-scores $\leq-2$ mean a significant low protein expression.

On the other hand, the Human Protein Atlas (https://www.proteinatlas.org/) explains the diverse molecular signatures of proteomes in human tissues based on an integrated 'omics' approach that involves quantitative transcriptomics and tissue microarray-based immunohistochemistry ${ }^{58,88,115}$. We compared the protein expression levels (high, medium, low and non-detected) of our 230 proteins between normal and BC tissues. Finally, all genes with the altered protein expression made up the third OncoOmics approach.

Breast cancer dependency map. The DepMap project (https://depmap.org/portal/) is collaboration between the Broad Institute and the Welcome Sanger Institute. Multiple genetic or epigenetic changes provide cancer cells with specific vulnerabilities that normal cells lack. Even though the landscape of genomic alterations has been extensively studied to date, we have limited understanding of the biological impact of these alterations in the development of specific tumor vulnerabilities, which triggers a limited use of precision medicine in the clinical practice worldwide. Therefore, the main goal of DepMap is to create a comprehensive preclinical reference map connecting tumor features with tumor dependencies to accelerate the development of precision treatments ${ }^{19-22}$.

In order to identify essential genes for BC cell proliferation and survival, DepMap performed systematic loss-of-function screens in a large number of well-annotated BC cell lines representing the tumor heterogeneity and their molecular subtypes. The DEMETER2 algorithm was applied to analyze genome-scale RNAi loss-of-function screens in $73 \mathrm{BC}$ cell lines and 711 cancer cell lines, whereas the CERES algorithm was applied to analyze genome-scale CRISPR-Cas9 loss-of-function screens in $28 \mathrm{BC}$ cell lines and 558 cancer cell lines ${ }^{20,22}$. In addition to existing cell lines, the Cancer Cell Line Encyclopedia (CCLE) project will greatly expand the collection of characterized cell lines to improve precision treatments ${ }^{116}$.

Regarding dependency scores, a lower score means that a gene is more likely to be dependent in a specific cancer cell line. A score of 0 means that a gene is not essential, whereas a score of -1 corresponds to the median of all common essential genes. A strongly selective gene means that its dependency is at least 100 times more likely to have been sampled from a skewed distribution than a normal distribution. A common essential gene is when in a pan-cancer screen its gene ranks in the top most depleting genes in at least $90 \%$ of cell lines ${ }^{19}$. All genes or proteins with a dependency score $\leq-1$ were subsequently analyzed with patient-derived xenografts.

Patient-derived xenografts. The Jackson Laboratory PDX resource (http://tumor.informatics.jax.org/ mtbwi/pdxSearch.do) comprises 455 PDX models originating from 34 different primary sites ${ }^{59}$. Even though, we analyzed expression levels of strongly selective and common essential proteins in breast cancer obtained from the analysis of BC dependency map in cell lines. Significant high protein expression has a Z-score $\geq 2$ and significant low protein expression has a Z-scores $\leq-2$. 
Enrichment map of the OncoOmics BC essential genes. The pathway enrichment analysis gives scientists curated interpretation of gene lists generated from genome-scale experiments ${ }^{66}$. The OncoOmics essential genes in BC were analyzed by using g:Profiler (https://biit.cs.ut.ee/gprofiler/) in order to obtain significant annotations (FDR < 0.001) related to GO terms, pathways, networks and disease phenotypes. Subsequently, g:Profiler annotations were analyzed with the EnrichmentMap software in order to generate network interactions of the most relevant GO: biological processes and Reactome pathways, and these networks were visualized using Cytoscape $\mathrm{e}^{54,66}$.

Clinical trials. The Open Targets Platform (https://www.targetvalidation.org) is comprehensive and robust data integration for access to and visualization of drugs involved in clinical trials associated with BC proteins, detailing its phase, status, type and target class ${ }^{69}$. In addition, we created a Sankey plot to better understand which drugs are involved in the most advanced phases ( 3 and 4 ) of clinical trials.

Precision medicine. Precision oncology focuses on matching the most effective treatment based on the 'omics' profile of each individual or population ${ }^{70,71}$. The CGI (https://www.cancergenomeinterpreter.org/home) flags genomic biomarkers of drug response with different levels of clinical relevance ${ }^{38}$. Huang et al. and the Pan-Cancer Atlas project conducted the largest investigation of pathogenic germline variants in cancer ${ }^{73}$. Long et al. ${ }^{74,75}$, Cai et al. ${ }^{76}$, and Michailidou et al.$^{77}$, performed genome-wide association studies identifying germline variations related to BC development. PreMedKB (http://www.fudan-pgx.org/premedkb/index.html\#/home) is a bioinformatics tool that facilitates the interpretation of the clinical meaning of a patient's genetic variants ${ }^{71}$. PharmGKB (https://www.pharmgkb.org/) collected complete guidelines for application of pharmacogenomics in clinical practice, according to several consortiums worldwide ${ }^{43-46}$. Finally, PCAWG Consortium (https://dcc.icgc. $\mathrm{org} /$ ) revealed an integrative analysis of genomic alterations in coding and non-coding regions ${ }^{6,92}$

Based on the aforementioned somatic and germline oncogenic variants we performed two analyses. On the one hand, we analyzed the consequence type of variants with the Ensembl Variant Effector Predictor (https:// www.ensembl.org/Multi/Tools/VEP? $\mathrm{db}=$ core), which is a powerful toolset for the annotation of genomic variants in coding and non-coding regions ${ }^{80}$. On the other hand, we analyzed oncogenic variants through the Cancer Genome Interpreter and PreMedKB platforms to provide a comprehensive in silico list of biological therapy $\operatorname{drugs}^{38,71}$.

Statistical analyses. We performed a multiple comparison using the Bonferroni correction test (significant level of $\mathrm{P}<0.05$ and a $95 \%$ confidence interval) to analyze: 1) significant differences of genomic alteration frequencies among non-cancer genes, BC driver genes, Consensus Strategy, Pan-Cancer Atlas and PharmGKB/CGI genes; 2) significant differences of genomic alteration frequencies among intrinsic molecular subtypes and tumor stages; 3 ) significant differences of genomic alteration frequencies of signaling pathways among molecular subtypes and tumor stages. A significant correlation of the degree centrality between the String PPi network and the OncoPPi BC network was performed using the Spearman $\mathrm{p}$-value test with a $\mathrm{P}<0.05$. The significant high and low protein expression in humn tissues and patient-derived xenografts was considered using the Z-score. Z-score $\geq 2$ means significant high protein expression and Z-scores $\leq-2$ means significant low protein expression. Lastly, the enrichment map of OncoOmics BC essential genes was performed using g:Profiler that determines the most significant GO: biological processes, GO: molecular functions, Reactome pathways, WikiPathways, KEGG pathways and human phenotype ontology with a false discovery rate $<0.001$.

\section{Data availability}

All data generated or analyzed during this study are included in this published article (and its Supplementary Information files).

Received: 15 July 2019; Accepted: 2 March 2020;

Published online: 24 March 2020

\section{References}

1. Espinal-Enríquez, J., Fresno, C., Anda-Jáuregui, G. \& Hernández-Lemus, E. RNA-Seq based genome-wide analysis reveals loss of inter-chromosomal regulation in breast cancer. Sci. Rep. 7, 1760 (2017).

2. Guerrero, S. et al. Analysis of Racial/Ethnic Representation in Select Basic and Applied Cancer Research Studies. 1-8. https://doi. org/10.1038/s41598-018-32264-x (2018).

3. Ding, L. et al. Perspective on Oncogenic Processes at the End of the Beginning of Cancer Genomics. Cell 173, 305-320.e10 (2018).

4. Bray, F. et al. Global cancer statistics 2018: GLOBOCAN estimates of incidence and mortality worldwide for 36 cancers in 185 countries. CA. Canc er J. Clin. https://doi.org/10.3322/caac.21492 (2018).

5. Sjöblom, T. et al. The consensus coding sequences of human breast and colorectal cancers. Science (80-.). https://doi.org/10.1126/ science.1133427 (2006).

6. Rheinbay, E., Nielsen, M. M., Abascal, F. \& Wala, J. A. Analyses of non-coding somatic drivers in 2, 658 cancer whole genomes. Nature. https://doi.org/10.1038/s41586-020-1965-x (2020).

7. Kandoth, C. et al. Mutational landscape and significance across 12 major cancer types. Nature. https://doi.org/10.1038/nature12634 (2013).

8. Lawrence, M. S. et al. Discovery and saturation analysis of cancer genes across 21 tumour types. Nature. https://doi.org/10.1038/ nature12912 (2014)

9. Porta-Pardo, E. et al. Comparison of algorithms for the detection of cancer drivers at subgene resolution. Nat. Methods. https://doi. org/10.1038/nmeth.4364 (2017).

10. Tamborero, D. et al. Comprehensive identification of mutational cancer driver genes across 12 tumor types. Sci. Rep. 3, 2650 (2013).

11. Lu, C. et al. Patterns and functional implications of rare germline variants across 12 cancer types. Nat. Commun. https://doi. org/10.1038/ncomms10086 (2015).

12. Klijn, C. et al. A comprehensive transcriptional portrait of human cancer cell lines. Nat. Biotechnol. https://doi.org/10.1038/ nbt.3080 (2015). 
13. Gao, Q. et al. Driver Fusions and Their Implications in the Development and Treatment of Human Cancers. Cell Rep. https://doi. org/10.1016/j.celrep.2018.03.050 (2018).

14. Oltean, S. \& Bates, D. O. Hallmarks of alternative splicing in cancer. Oncogene. https://doi.org/10.1038/onc.2013.533 (2014).

15. Stricker, T. P. et al. Robust stratification of breast cancer subtypes using differential patterns of transcript isoform expression. PLoS Genet. https://doi.org/10.1371/journal.pgen.1006589 (2017).

16. Lawrence, R. T. et al. The Proteomic Landscape of Triple-Negative Breast Cancer. Cell Rep., https://doi.org/10.1016/j. celrep.2015.03.050 (2015).

17. Sogawa, K. et al. Identification of a novel serum biomarker for pancreatic cancer, C4b-binding protein $\alpha$-chain (C4BPA) by quantitative proteomic analysis using tandem mass tags. Br. J. Cancer. https://doi.org/10.1038/bjc.2016.295(2016).

18. Rubio-Perez, C. et al. In Silico Prescription of Anticancer Drugs to Cohorts of 28 Tumor Types Reveals Targeting Opportunities. Cancer Cell 27, 382-396 (2015).

19. Tsherniak, A. et al. Defining a Cancer Dependency Map. Cell. https://doi.org/10.1016/j.cell.2017.06.010 (2017)

20. Meyers, R. M. et al. Computational correction of copy number effect improves specificity of CRISPR-Cas9 essentiality screens in cancer cells. Nat. Genet. https://doi.org/10.1038/ng.3984 (2017).

21. Stransky, N. et al. Pharmacogenomic agreement between two cancer cell line data sets. Nature. https://doi.org/10.1038/nature15736 (2015).

22. McFarland, J. M. et al. Improved estimation of cancer dependencies from large-scale RNAi screens using model-based normalization and data integration. Nat. Commun. https://doi.org/10.1038/s41467-018-06916-5 (2018).

23. Shah, P. et al. Integrated Proteomic and Glycoproteomic Analyses of Prostate Cancer Cells Reveal Glycoprotein Alteration in Protein Abundance and Glycosylation. Mol. Cell. Proteomics. https://doi.org/10.1074/mcp.M115.047928 (2015).

24. Bernard, P. S. et al. Supervised risk predictor of breast cancer based on intrinsic subtypes. J. Clin. Oncol. 27, 1160-1167 (2009).

25. Kumar, R., Sharma, A. \& Tiwari, R. K. Application of microarray in breast cancer: An overview. J. Pharm. Bioallied Sci. 4, 21-6 (2012).

26. Banerji, S. et al. Sequence analysis of mutations and translocations across breast cancer subtypes. Nature 486, 405-409 (2012)

27. López-Cortés, A. et al. Breast cancer risk associated with gene expression and genotype polymorphisms of the folate-metabolizing MTHFR gene: a case-control study in a high altitude Ecuadorian mestizo population. Tumor Biol. 36, 6451-6461 (2015).

28. Prat, A., Ellis, M. J. \& Perou, C. M. Practical implications of gene-expression-based assays for breast oncologists. Nature Reviews Clinical Oncology 9, 48-57 (2012).

29. López-Cortés, A. et al. Gene prioritization, communality analysis, networking and metabolic integrated pathway to better understand breast cancer pathogenesis. Sci. Rep. 8, 16679 (2018).

30. López-cortés, A. et al. Mutational Analysis of Oncogenic AKT1 Gene Associated with Breast Cancer Risk in the High Altitude Ecuadorian Mestizo Population. 2018 (2018).

31. Huang, K. L. et al. Pathogenic Germline Variants in 10,389 Adult Cancers. Cell 355-370, https://doi.org/10.1016/j.cell.2018.03.039 (2018).

32. Bailey, M. H. et al. Comprehensive Characterization of Cancer Driver Genes and Mutations. Cell 173, 371-385.e18 (2018).

33. Thorsson, V. et al. The Immune Landscape of Cancer. Immunity 1-19. https://doi.org/10.1016/j.immuni.2018.03.023 (2018).

34. Liu, J. et al. An Integrated TCGA Pan-Cancer Clinical Data Resource to Drive High-Quality Survival Outcome Analytics. Cell. https://doi.org/10.1016/j.cell.2018.02.052 (2018).

35. Sanchez-Vega, F. et al. Oncogenic Signaling Pathways in The Cancer Genome Atlas. Cell 173, 321-337.e10 (2018).

36. Hoadley, K. A. et al. Cell-of-Origin Patterns Dominate the Molecular Classification of 10,000 Tumors from 33 Types of Cancer. Cell 173, 291-304.e6 (2018).

37. Ellrott, K. et al. Scalable Open Science Approach for Mutation Calling of Tumor Exomes Using Multiple Genomic Pipelines. Cell Syst. https://doi.org/10.1016/j.cels.2018.03.002 (2018).

38. Tamborero, D. et al. Cancer Genome Interpreter annotates the biological and clinical relevance of tumor alterations. 1-8. https:// doi.org/10.1186/s13073-018-0531-8 (2018).

39. Thorn, C. F., Klein, T. E. \& Altman, R. B. PharmGKB: The pharmacogenomics knowledge base. Methods Mol. Biol. 1015, 311-320 (2013).

40. Tejera, E. et al. Consensus strategy in genes prioritization and combined bioinformatics analysis for preeclampsia pathogenesis. BMC Med. Genomics 10, 50 (2017).

41. Cabrera-andrade, A. Gene Prioritization through Consensus Strategy, Enrichment Methodologies Analysis, and Networking for Osteosarcoma Pathogenesis. Int. J. Mol. Sci. 21, 1-21 (2020).

42. Barbarino, J. M., Whirl-Carrillo, M., Altman, R. B. \& Klein, T. E. PharmGKB: A worldwide resource for pharmacogenomic information. Wiley Interdisciplinary Reviews: Systems Biology and Medicine. https://doi.org/10.1002/wsbm.1417 (2018).

43. Ross, C. J. D. et al. The Canadian Pharmacogenomics Network for Drug Safety: a model for safety pharmacology. Thyroid 20, 681-7 (2010).

44. Saito, Y. et al. CPIC: Clinical Pharmacogenetics Implementation Consortium of the Pharmacogenomics Research Network. Clinical Pharmacology and Therapeutics 99, 36-37 (2016).

45. Swen, J. J. et al. Pharmacogenetics: From bench to byte an update of guidelines. Clin. Pharmacol. Ther. 89, 662-673 (2011).

46. European Society for Medicinal Oncology. Breast Cancer: A guide for patients. European Society for Medical Oncology (2018).

47. Cerami, E. et al. The cBio cancer genomics portal: an open platform for exploring multidimensional cancer genomics data. Cancer Discov. https://doi.org/10.1158/2159-8290.CD-12-0095 (2012).

48. Gao, J. et al. Integrative analysis of complex cancer genomics and clinical profiles using the cBioPortal. Sci. Signal. https://doi. org/10.1126/scisignal.2004088 (2013).

49. Huang, D. W., Sherman, B. T. \& Lempicki, R. A. Systematic and integrative analysis of large gene lists using DAVID bioinformatics resources. Nat. Protoc. 4, 44-57 (2009).

50. Huang, D. W., Sherman, B. T. \& Lempicki, R. A. Bioinformatics enrichment tools: paths toward the comprehensive functional analysis of large gene lists. Nucleic Acids Res. 37, 1-13 (2009).

51. Antonov, A. V., Schmidt, E. E., Dietmann, S., Krestyaninova, M. \& Hermjakob, H. R spider: a network-based analysis of gene lists by combining signaling and metabolic pathways from Reactome and KEGG databases. Nucleic Acids Res. 38, W78-83 (2010).

52. Ogata, H. et al. KEGG: Kyoto encyclopedia of genes and genomes. Nucleic Acids Research 27, 29-34 (1999).

53. Szklarczyk, D. et al. STRING v10: protein-protein interaction networks, integrated over the tree of life. Nucleic Acids Res. 43, D447-52 (2015)

54. Shannon, P. et al. Cytoscape: a software environment for integrated models of biomolecular interaction networks. Genome Res. 13, 2498-504 (2003).

55. Ivanov, A. A. et al. The OncoPPi Portal: an integrative resource to explore and prioritize protein-protein interactions for cancer target discovery. Bioinformatics 1-9. https://doi.org/10.1093/bioinformatics/btx743 (2017).

56. Li, Z. et al. The OncoPPi network of cancer-focused protein-protein interactions to inform biological insights and therapeutic strategies. Nat. Commun. 8 (2017).

57. Uhlen, M. et al. A pathology atlas of the human cancer transcriptome. Science (80-.). https://doi.org/10.1126/science.aan2507 (2017).

58. Uhlén, M. et al. Tissue-based map of the human proteome. Science (80-.)., https://doi.org/10.1126/science.1260419 (2015). 
59. Woo, X. Y. et al. Genomic data analysis workflows for tumors from patient-derived xenografts (PDXs): Challenges and guidelines. BMC Med. Genomics. https://doi.org/10.1186/s12920-019-0551-2 (2019).

60. Repana, D. et al. The Network of Cancer Genes (NCG): a comprehensive catalogue of known and candidate cancer genes from cancer sequencing screens. Genome Biol. https://doi.org/10.1186/s13059-018-1612-0 (2019).

61. Patel, S. J. et al. Identification of essential genes for cancer immunotherapy. Nature. https://doi.org/10.1038/nature23477 (2017).

62. Manning, G., Whyte, D. B., Martinez, R., Hunter, T. \& Sudarsanam, S. The protein kinase complement of the human genome. Science. https://doi.org/10.1126/science.1075762 (2002).

63. Bar-Joseph, Z. et al. Genome-wide transcriptional analysis of the human cell cycle identifies genes differentially regulated in normal and cancer cells. Proc. Natl. Acad. Sci. https://doi.org/10.1073/pnas.0704723105 (2008).

64. Chae, Y. K. et al. Genomic landscape of DNA repair genes in cancer. Oncotarget. https://doi.org/10.18632/oncotarget.8196 (2016).

65. Hentze, M. W., Castello, A., Schwarzl, T. \& Preiss, T. A brave new world of RNA-binding proteins. Nature Reviews Molecular Cell Biology. https://doi.org/10.1038/nrm.2017.130 (2018).

66. Reimand, J. et al. Pathway enrichment analysis and visualization of omics data using g:Profiler, GSEA, Cytoscape and EnrichmentMap. Nat. Protoc. https://doi.org/10.1038/s41596-018-0103-9 (2019).

67. Fabregat, A. et al. The Reactome Pathway Knowledgebase. Nucleic Acids Res. https://doi.org/10.1093/nar/gkx1132 (2018).

68. Posey, J. E. et al. Resolution of Disease Phenotypes Resulting from Multilocus Genomic Variation. N. Engl. J. Med. https://doi. org/10.1056/nejmoa1516767 (2016).

69. Carvalho-Silva, D. et al. Open Targets Platform: New developments and updates two years on. Nucleic Acids Res. https://doi. org/10.1093/nar/gky1133 (2019)

70. Shin, S. H., Bode, A. M. \& Dong, Z. Precision medicine: the foundation of future cancer therapeutics. npj Precis. Oncol. https://doi. org/10.1038/s41698-017-0016-Z (2017)

71. Yu, Y. et al. PreMedKB: An integrated precision medicine knowledgebase for interpreting relationships between diseases, genes, variants and drugs. Nucleic Acids Res. https://doi.org/10.1093/nar/gky1042 (2019).

72. Arnedos, M. et al. Precision medicine for metastatic breast cancer-limitations and solutions. Nature Reviews Clinical Oncology. https://doi.org/10.1038/nrclinonc.2015.123 (2015).

73. Huang, K. lin et al. Pathogenic Germline Variants in 10,389 Adult Cancers. Cell. https://doi.org/10.1016/j.cell.2018.03.039 (2018).

74. Long, J. et al. Genome-wide association study in East Asians identifies novel susceptibility loci for breast cancer. PLoS Genet. https://doi.org/10.1371/journal.pgen.1002532 (2012).

75. Long, J. et al. A common deletion in the APOBEC3 genes and breast cancer risk. J. Natl. Cancer Inst. https://doi.org/10.1093/jnci/

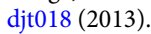

76. Cai, Q. et al. Genome-wide association analysis in East Asians identifies breast cancer susceptibility loci at 1q32.1, 5q14.3 and 15q26.1. Nat. Genet. https://doi.org/10.1038/ng.3041 (2014).

77. Michailidou, K. et al. Association analysis identifies 65 new breast cancer risk loci. Nature. https://doi.org/10.1038/nature24284 (2017).

78. Whirl-Carrillo, M. et al. Pharmacogenomics knowledge for personalized medicine. Clinical Pharmacology and Therapeutics. https://doi.org/10.1038/clpt.2012.96 (2012).

79. Amstutz, U. et al. Clinical Pharmacogenetics Implementation Consortium (CPIC) Guideline for Dihydropyrimidine Dehydrogenase Genotype and Fluoropyrimidine Dosing: 2017 Update. Clin. Pharmacol. Ther. 103, 210-216 (2018).

80. McLaren, W. et al. The Ensembl Variant Effect Predictor. Genome Biol. https://doi.org/10.1186/s13059-016-0974-4 (2016).

81. Thomas, S. J., Snowden, J. A., Zeidler, M. P. \& Danson, S. J. The role of JAK/STAT signalling in the pathogenesis, prognosis and treatment of solid tumours. Br. J. Cancer. https://doi.org/10.1038/bjc.2015.233 (2015).

82. Kazi, M., Trivedi, T., Kobawala, T. \& Ghosh, N. The Potential of Wnt Signaling Pathway in Cancer: A Focus on Breast Cancer. Cancer Transl. Med. https://doi.org/10.4103/2395-3977.181437 (2016).

83. Xie, B. et al. Benzyl Isothiocyanate potentiates $\mathrm{p} 53$ signaling and antitumor effects against breast cancer through activation of p53LKB1 and p73-LKB1 axes. Sci. Rep. 7 (2017).

84. Paz-y-Miño, C. et al. Incidence of the L858R and G719S mutations of the epidermal growth factor receptor oncogene in an Ecuadorian population with lung cancer. Cancer Genet. Cytogenet. 196 (2010).

85. Wu, L. \& Yang, X. Targeting the Hippo Pathway for Breast Cancer Therapy. Cancers (Basel). https://doi.org/10.3390/ cancers10110422 (2018)

86. Uzair, I. D., Conte Grand, J., Flamini, M. I. \& Sanchez, A. M. Molecular Actions of Thyroid Hormone on Breast Cancer Cell Migration and Invasion via Cortactin/N-WASP. Front. Endocrinol. (Lausanne). https://doi.org/10.3389/fendo.2019.00139 (2019).

87. Ivanov, A. A. et al. The OncoPPi Portal: An integrative resource to explore and prioritize protein-protein interactions for cancer target discovery. Bioinformatics. https://doi.org/10.1093/bioinformatics/btx743 (2018).

88. Uhlén, M. et al. A Human Protein Atlas for Normal and Cancer Tissues Based on Antibody Proteomics. Mol. Cell. Proteomics. https://doi.org/10.1074/mcp.M500279-MCP200 (2005).

89. Koboldt, D. C. et al. Comprehensive molecular portraits of human breast tumours. Nature 490, 61-70 (2012).

90. Murayama \& Gotoh. Patient-Derived Xenograft Models of Breast Cancer and Their Application. Cells. https://doi.org/10.3390/ cells8060621 (2019).

91. Gonzalez-Perez, A. et al. IntOGen-mutations identifies cancer drivers across tumor types. Nat. Methods 10, 1081-1082 (2013).

92. ICGC/TCGA Pan-Cancer Analysis of Whole Genomes Consortium. Pan-cancer analysis of whole genomes. Nature. https://doi. org/10.1038/s41586-020-1969-6 (2020)

93. De Mattos-Arruda, L. et al. The Genomic and Immune Landscapes of Lethal Metastatic Breast Cancer. Cell Rep. 27, 2690-2708.e10 (2019).

94. Adams, S. et al. Current Landscape of Immunotherapy in Breast Cancer. JAMA Oncol. 1-10. https://doi.org/10.1001/ jamaoncol.2018.7147 (2019)

95. Lopez-Cortes, A. et al. Prediction of breast cancer proteins using molecular descriptors and artificial neural networks: a focus on cancer immunotherapy proteins, metastasis driver proteins, and RNA-binding proteins. bioRxiv Bioinforma., https://doi. org/10.1101/840108 (2019).

96. López-Cortés, A. et al. Prediction of druggable proteins using machine learning and functional enrichment analysis: a focus on cancer-related proteins and RNA-binding proteins. bioRxiv. https://doi.org/10.1101/825513 (2019).

97. Miller, S. M., Goulet, D. R. \& Johnson, G. L. Targeting the Breast Cancer Kinome. J. Cell. Physiol. https://doi.org/10.1002/jcp.25427 (2017).

98. Caldon, C. E., Daly, R. J., Sutherland, R. L. \& Musgrove, E. A. Cell cycle control in breast cancer cells. Journal of Cellular Biochemistry. https://doi.org/10.1002/jcb.20690 (2006).

99. Majidinia, M. \& Yousefi, B. DNA repair and damage pathways in breast cancer development and therapy. DNA Repair. https://doi. org/10.1016/j.dnarep.2017.03.009 (2017).

100. Pereira, B., Billaud, M. \& Almeida, R. RNA-Binding Proteins in Cancer: Old Players and New Actors. Trends in Cancer. https://doi. org/10.1016/j.trecan.2017.05.003(2017).

101. Wurth, L. et al. UNR/CSDE1 Drives a Post-transcriptional Program to Promote Melanoma Invasion and Metastasis. Cancer Cell 30, 694-707 (2016). 
102. Guerrero, S. et al. In silico analyses reveal new putative Breast Cancer RNA-binding proteins. bioRxiv. https://doi. org/10.1101/2020.01.08.898965 (2020).

103. Kechavarzi, B. \& Janga, S. C. Dissecting the expression landscape of RNA-binding proteins in human cancers. Genome Biol. https:// doi.org/10.1186/gb-2014-15-1-r14 (2014).

104. Wang, J., Liu, Q. \& Shyr, Y. Dysregulated transcription across diverse cancer types reveals the importance of RNA-binding protein in carcinogenesis. BMC Genomics. https://doi.org/10.1186/1471-2164-16-S7-S5 (2015).

105. Sebestyén, E. et al. Large-scale analysis of genome and transcriptome alterations in multiple tumors unveils novel cancer-relevant splicing networks. Genome Res. https://doi.org/10.1101/gr.199935.115 (2016).

106. García-cárdenas, J. M. et al. Post-transcriptional Regulation of Colorectal Cancer: A Focus on RNA-Binding. Proteins. 6, 1-18 (2019).

107. López-Cortés, A. et al. Pharmacogenomics, biomarker network, and allele frequencies in colorectal cancer. Pharmacogenomics J. https://doi.org/10.1038/s41397-019-0102-4 (2019).

108. Harris, E. E. R. Precision Medicine for Breast Cancer: The Paths to Truly Individualized Diagnosis and Treatment. Int. J. Breast Cancer, https://doi.org/10.1155/2018/4809183 (2018).

109. López-Cortés, A., Guerrero, S., Redal, M. A., Alvarado, A. T. \& Quiñones, L. A. State of art of cancer pharmacogenomics in Latin American populations. International Journal of Molecular Sciences 18 (2017)

110. Quinones, L. et al. Perception of the Usefulness of Drug/Gene Pairs and Barriers for Pharmacogenomics in Latin America. Curr. Drug Metab. 15, 202-208 (2014).

111. Beroukhim, R. et al. The landscape of somatic copy-number alteration across human cancers. Nature. https://doi.org/10.1038/ nature 08822 (2010).

112. Mermel, C. H. et al. GISTIC2.0 facilitates sensitive and confident localization of the targets of focal somatic copy-number alteration in human cancers. Genome Biol. https://doi.org/10.1186/gb-2011-12-4-r41 (2011).

113. Rocco, P. et al. OncoScore: A novel, Internet-based tool to assess the oncogenic potential of genes. Sci. Rep. https://doi.org/10.1038/ srep46290 (2017).

114. Berger, A. C. et al. A Comprehensive Pan-Cancer Molecular Study of Gynecologic and Breast Cancers. Cancer Cell 1-16. https:// doi.org/10.1016/j.ccell.2018.03.014 (2018).

115. Uhlen, M. et al. Towards a knowledge-based Human Protein Atlas. Nat. Biotechnol. https://doi.org/10.1038/nbt1210-1248 (2010).

116. Barretina, J. et al. The Cancer Cell Line Encyclopedia enables predictive modelling of anticancer drug sensitivity. Nature 483, 603-7 (2012).

\section{Acknowledgements}

This work was supported by Universidad UTE (Ecuador), Universidad de Las Américas (Ecuador), University of A Coruna (Spain), University of the Basque Country (Spain), and McGill University (Canada). Additionally, this work was supported by "Collaborative Project in Genomic Data Integration (CICLOGEN)" PI17/01826 funded by the Carlos III Health Institute from the Spanish National plan for Scientific and Technical Research and Innovation 2013-2016 and the European Regional Development Funds (FEDER).

\section{Author contributions}

A.L.C. conceived the subject, the conceptualization of the study and wrote the manuscript. E.T., S.J.B., C.R.M., H.G.D. and C.P.y.M. supervised the project. A.L.C. and C.P.y.M. did founding acquisition. A.L.C., S.G. and A.C.A. did data curation and supplementary data. E.T., S.G., A.C.A., S.J.B., C.R.M., H.G.D., A.P., Y.P.C. and C.P.y.M. gave conceptual advice and valuable scientific input. Finally, all authors reviewed the manuscript.

\section{Competing interests}

The authors declare no competing interests.

\section{Additional information}

Supplementary information is available for this paper at https://doi.org/10.1038/s41598-020-62279-2.

Correspondence and requests for materials should be addressed to A.L.-C. or E.T.

Reprints and permissions information is available at www.nature.com/reprints.

Publisher's note Springer Nature remains neutral with regard to jurisdictional claims in published maps and institutional affiliations.

Open Access This article is licensed under a Creative Commons Attribution 4.0 International License, which permits use, sharing, adaptation, distribution and reproduction in any medium or format, as long as you give appropriate credit to the original author(s) and the source, provide a link to the Creative Commons license, and indicate if changes were made. The images or other third party material in this article are included in the article's Creative Commons license, unless indicated otherwise in a credit line to the material. If material is not included in the article's Creative Commons license and your intended use is not permitted by statutory regulation or exceeds the permitted use, you will need to obtain permission directly from the copyright holder. To view a copy of this license, visit http://creativecommons.org/licenses/by/4.0/.

(C) The Author(s) 2020 